\title{
Multiobjective Environmentally Sustainable Optimal Design of Dedicated Connected Autonomous Vehicle Lanes
}

\author{
Yu Lin ${ }^{1}$, Hongfei Jia ${ }^{1, *}$, Bo Zou ${ }^{2} \mathbb{D}$, Hongzhi Miao ${ }^{3} \mathbb{D}$, Ruiyi $\mathrm{Wu}^{1}{ }^{1}$, Jingjing Tian ${ }^{1}$ and Guanfeng Wang ${ }^{1}$ \\ 1 Transportation College, Jilin University, Changchun 130022, China; yulin735@gmail.com (Y.L.); \\ wury18@mails.jlu.edu.cn (R.W.); tianjj19@mails.jlu.edu.cn (J.T.); wanggf20@mails.jlu.edu.cn (G.W.) \\ 2 Department of Civil, Materials, and Environmental Engineering, University of Illinois at Chicago, \\ Chicago, IL 60607, USA; bzou@uic.edu \\ 3 College of Transportation Engineering, Dalian Maritime University, Dalian 116026, China; \\ honmgee@foxmail.com \\ * Correspondence: jiahf@jlu.edu.cn
}

Citation: Lin, Y.; Jia, H.; Zou, B.; Miao, H.; Wu, R.; Tian, J.; Wang, G. Multiobjective Environmentally Sustainable Optimal Design of Dedicated Connected Autonomous Vehicle Lanes. Sustainability 2021, 13, 3454. https://doi.org/10.3390/ su13063454

Academic Editor: Itzhak Benenson

Received: 23 February 2021

Accepted: 12 March 2021

Published: 20 March 2021

Publisher's Note: MDPI stays neutral with regard to jurisdictional claims in published maps and institutional affiliations.

Copyright: (c) 2021 by the authors. Licensee MDPI, Basel, Switzerland. This article is an open access article distributed under the terms and conditions of the Creative Commons Attribution (CC BY) license (https:/ / creativecommons.org/licenses/by/ $4.0 /)$.

\begin{abstract}
The emergence of connected autonomous vehicles (CAVs) is not only improving the efficiency of transportation, but also providing new opportunities for the sustainable development of transportation. Taking advantage of the energy consumption of CAVs to promote the sustainable development of transportation has attracted extensive public attention in recent years. This paper develops a mathematical approach to investigating the problem of the optimal implementation of dedicated CAV lanes while simultaneously considering economic and environmental sustainability. Specifically, the problem is described as a multi-objective bi-level programming model, in which the upper level is to minimize the system-level costs including travel time costs, CAV lane construction cost, and emission cost, whereas the lower level characterizes the multi-class network equilibrium with a heterogeneous traffic stream consisting of both human-driven vehicle (HVs) and CAVs. To address the multi-objective dedicated CAV lane implement problem, we propose an integrated solution framework that integrates a non-dominated sorting genetic algorithm II (NSGA-II) algorithm, diagonalized algorithm, and Frank-Wolfe algorithm. The NSGA-II was adopted to solve the upperlevel model, i.e., hunting for the optimal CAV lanes implementation schemes. The diagonalized FrankWolfe (DFW) algorithm is used to cope with multi-class network equilibrium. Finally, numerical experiments were conducted to demonstrate the effectiveness of the proposed model and solution method. The experimental results show that the total travel time cost, total emission cost, and total energy consumption were decreased by about $12.03 \%, 10.42 \%$, and $9.4 \%$, respectively, in the Nguyen-Dupuis network as a result of implementing the dedicated CAV lanes.
\end{abstract}

Keywords: sustainable transportation; connected autonomous vehicle lane; heterogeneous traffic streams; multi-class network equilibrium

\section{Introduction}

Emissions from transportation systems are considered to be an important component of environmental pollutants. With the expansion of transportation systems and road facilities, emissions from road traffic have been increasing over the years, and the environmental impacts of traffic emissions are receiving increasing attention from researchers [1]. The sustainable development of road transportation in terms of low emissions of carbon dioxide $\left(\mathrm{CO}_{2}\right)$ and criteria pollutants is becoming a requirement and challenge for the transportation of planning designers. Although many measures for alleviating the impact of traffic on the environment (e.g., the adoption of alternative energy vehicles) have been developed, traffic planners should propose solutions with a broader perspective, such as through road network design [2]. To this end, it is critical to quantify the impact of road traffic on the environment when designing transportation networks, not only with the aim of minimizing the system travel time (or associated cost), but also seeking to minimize the 
negative impacts of traffic on the environment $[3,4]$. Some researchers have investigated transportation network design problems (NDPs) in the context of environmental considerations, such as minimizing emission and traffic noise from different perspectives, but to our knowledge not in a context involving a heterogeneous traffic stream consisting of connected and autonomous vehicles (CAVs) and human-driven vehicles (HVs) $[5,6]$.

CAVs have been undergoing rapid development [7-9]. It has been widely accepted that CAV use will be a part of future transportation systems. On the other hand, the transformation from HVs to CAVs will not be accomplished overnight. Instead, a gradual development process is expected. As such, heterogeneous traffic streams consisting of CAVs and HVs will likely persist for a long time. Compared to HVs, the use of CAVs leads to many benefits to the transportation system, including improvements in road capacity $[10,11]$ and traffic operation $[12,13]$, as well as reduced emissions $[9,14]$ and enhanced road safety $[15,16]$. In particular, compared to HVs, CAVs are estimated to reduce emissions by $20-50 \%$, thus offering huge potential for the promotion of sustainable transportation $[17,18]$. Road traffic driving conditions affect the benefits of CAVs, because the intelligent driving of CAVs is dependent on communications equipment and roadside facilities. Some scholars have proposed implementing dedicated CAV lanes in existing road networks to improve system efficiency by offering a better driving environment for CAVs, which would also reduce system travel time [19]. However, most existing studies only focus on the optimal travel time of the system. In other words, a multi-objective optimization approach that takes into account both system travel time and emissions is necessary to understand the best ways to implement CAV lanes. To the best of our knowledge, no such research exists in the literature.

To fill this gap in the study of sustainable dedicated CAV lane design, in this paper we consider a bi-level multi-objective mathematical programming approach that considers objectives related to both system efficiency and sustainability. The upper level of the proposed model seeks the optimal CAV lane implementation schemes taking into account system travel time cost and emission cost, while also minimizing CAV lane construction cost. The lower-level captures multi-class network equilibrium consisting of two classes of vehicles, i.e., $\mathrm{CAVs}$ and $\mathrm{HVs}$. To solve the bi-level multi-objective optimization model, an integrated solution framework combining the non-dominated sorting genetic algorithm II(NSGA-II) and diagonalized Frank-Wolfe (DFW) algorithm was developed. The NSGA-II algorithm is used to find the optimal CAV lane implementation schemes, while the DFW algorithm is used to solve multi-class network equilibrium at the lower-level. In this work, the multi-class network equilibrium addresses the heterogeneous traffic stream consisting of CAV and $\mathrm{HV}$, in which we assume that there needs to be no crisis effect between CAVs and HVs, because CAVs are regarded as HVs when driving in regular lanes. Additionally, we assume driving behavior (machine-to-human) to be ideal, without the consideration of driving crises. On the other hand, any potential crisis between CAVs is negligible when the CAVs are driving in dedicated CAV lanes, because CAVs are expected to experience better driving conditions as a result of their communication equipment and the roadside facilities provided by the CAV lanes.

Broadly speaking, sustainable dedicated CAV lane design belongs to the class of transportation network design problems. Such problems are inherently challenging to solve because: (1) the equilibrium constraints make the domain of feasible solutions nonconvex; (2) constructing the Pareto frontier for different objectives is a non-deterministic polynomial (NP) hard problem. In addition, solving multi-class (CAVs and HVs) network equilibrium needs dealing with the asymmetric impact on link cost between the two vehicle classes. Researchers convert multi-class network equilibrium to mixed-integer linear programs by non-linear complementary conditions in previous studies, which are then solved by solvers like CPLEX Optimizer [20,21]. However, solving such mixedinteger linear programs may still take a long time, because the equilibrium constraints make the domain of feasible solutions nonconvex. In this paper, we instead propose a diagonalized Frank-Wolfe algorithm, which is simple to implement and demonstrates 
good computational performance. On the other hand, we adopted the NSGA-II algorithm to handle the multi-objective and NP hard nature of the problem, which presents a better way to handle multiple objectives with different count units than traditional means such as the weighting method. Another strength of NSGA-II is that it can provide a large number of Pareto solutions, which will be desired for decision-making.

Moreover, some findings are obtained based on numerical examples. First, implementing dedicated CAV lanes could reduce the total time cost, total emission cost, and total energy consumption by about $12.03 \%, 10.42 \%$, and $9.4 \%$ in the Nguyen-Dupuis network. Second, implementing dedicated CAV lanes could further reduce the total travel cost and emission cost with CAV market penetration evolution. Third, expanding the capacity of dedicated CAV lanes will benefit the system's performance. In detail, compared to two times of capacity, the three times reduce the total travel cost and total emission cost by $5.5 \%$ and $4.6 \%$, respectively, when the CAV market penetration is $50 \%$. Finally, the relation between minimizing the total travel time cost and total emission cost is not always consistent, which is affected by vehicle travel speeds. In a nutshell, the contribution of this work lies in the following aspects.

(1) The proposed sustainable dedicated CAV lanes design problem is a novel research issue since it considers economic and vehicle emissions to capture the optimal CAV lanes implement scheme.

(2) A multi-objective bi-level programming model with multi-class network equilibrium constraints was developed to draw sustainable dedicated CAV lanes design problems.

(3) An integrated solution framework combining the meta-heuristic algorithm and diagonalized Frank-Wolfe (DFW) algorithm was designed to solve the dedicated CAV lanes design problems. Numerical experiments are finally conducted to test the proposed solution algorithm on different sizes of networks.

The rest of this study is organized as follows. Section 2 reviews the literature on CAVs and transportation network optimization problems. The problem formulations and the multi-objective bi-level model are presented in Sections 3 and 4, respectively. Section 5 reports the detailed solution algorithm. Section 6 shows the numerical experiment. Finally, Section 7 summarizes the conclusions and suggestions for future research.

\section{Literature Review}

The relevant literature of this work is generalized into two categories. First, some studies on network equilibrium involving CAV are presented. Second, a group of studies of the transportation network optimization problem is introduced, which includes the sustainable transportation network design and dedicated CAV lanes design problem.

\subsection{Previous Research on CAVs}

CAV as an emerging technology is reshaping our travel mode choice and lifestyle. Numerous studies are presented to investigate CAV from different perspectives. Milakis et al. [22] drew an overall review on the potential impact of CAV technology on transportation and society. Bagloee et al. [23] proposed a mixed traffic assignment model to investigate the network performance, in which connected vehicles follow the system optimal principle and non-connected vehicles obey the user equilibrium. Levin and Boyles [24] developed a multi-class traffic assignment cell transmission model, which analyzes the road capacity increment brings by different CAV penetration. Zhang and Nie [25] discussed the optimalratio control scheme of CAVs that follows the system optimal principle under a context of mixed traffic flow. Jiang [26] proposes a bi-level model to investigate the optimal signal design problem. The upper-level model seeks the optimal signal timing design at the intersection and the lower-level model solves a network equilibrium problem involving CAVs and HVs. Chen et al. [27] built a linear program model considering the CAVs ratio to investigate the network system optimum performance.

Recently studies have been concerned with CAV driving behavior. Considering naturalistic driving patterns, Adornato et al. [28] analyzed the battery match and state of 
charge based on driving data of field operational tests (FOTs). Liang et al. [29] explore the connection between driver distraction and crash risk using 24 different algorithms based on naturalistic driving data. Rahwan et al. [30] point out that human-machine cobehavior is a complicated process, which is difficult to solve in problems involving CAVs. Bonnefon et al. [31] investigated the different algorithms for solving the social dilemma of CAVs between two choices. Balsa-Barreiro et al. [32] developed a new approach to expressing the actual accessibility within the region by distorting traditional maps.

\subsection{Sustainable Transportation Network Optimization Problem}

Transportation network design aims to determine the optimal road network construction and system efficiency by modifying the road network layout or improving the link capacity. Sustainable network design is attracting extensive attention as a highly significant research direction of network design. $\mathrm{Xu}$ et al. [6] made a comprehensive review of sustainable road network design. Sharma and Mathew [33] developed a multi-objective optimization model to optimize sustainable road network design, which considers the speed-dependent model emission function. Wang and Szeto [4] proposed a discrete bi-level model to solve sustainable road networks. In the bi-level model, vehicle emission and noise are simultaneously taken as the optimization objections. Yin and Lawphongpanich [34] incorporated the cost recovery, service level, environment, and equity into a bi-level multiobjective model to address the road toll pricing and capacity investment problem. Szeto and Lo [35] investigated the impact of road network design on equity over time in terms of social, economic, and environmental dimensions.

Implementing dedicated CAV lanes as a newly emerging strategy used to handle the heterogeneous traffic steams was firstly proposed by Chen et al. [36]. The overall problem was formulated as a bi-level model, in which a diffusion model was introduced to predict the CAV demand evolution with time. Chen et al. [21] build a two-stage model of the CAV lanes implement problem. The first stage model was adopted to find the optimal CAV lanes implement scheme. The second stage model was to solve multiclass network equilibrium involving CAVs and HVs. Liu and Song [37] incorporated the CAV lanes and toll lanes into a robust model to determine the optimal joint implement scheme. Chen et al. [38] developed a new idea allowing CAVs to drive on bus rapid transit (BRT) lanes to improve the utilization of BRT lanes, which prevented providing excessive dedicated CAV lanes.

\section{Problem Formulation}

\subsection{Network Representation}

Let $G(N, A)$ denote a general urban transportation network, where $N$ represents the set of the nodes and $A$ indicates the set of links. Define $\hat{A}$ as the CAV link sets of the network, and $\mathrm{K}$ as the sets of pairs including CAV links and HV links. For instance, Figure 1 shows a simple network to illustrate the process of CAV lanes implementation. The number on each edge represents the link number. Figure 1a shows the network without CAV links. After deploying CAV links, assuming that link 2 and link 3 are the candidate CAV links, thus, the updated network is Figure $1 b$, where $A=\left\{1,2,2^{\prime}, 3,3^{\prime}\right\}$ and $\hat{A}=\left\{2^{\prime}, 3^{\prime}\right\}$, and $K=\left\{\left(2,2^{\prime}\right),\left(3,3^{\prime}\right)\right\}$. To distinguish two types of vehicles, we defined $M=\{1,2\}$ to indicate the set of travel modes, where 1 represents the CAV mode and 2 represents the HV mode. Moreover, let $w \in W$ denote the origin-destination (OD) pairs. Define $q_{w}^{m}$ as the travel demand of OD pairs $w \in W$ for travel mode $m$. Let $R$ represents the path set and $\hat{R}$ denote the CAV path set.

Note that the CAV link includes at least one candidate CAV lane, but the link lanes will not all be converted to CAV lanes so as to guarantee the right of way of HVs. Only CAVs are allowed to drive on the dedicated CAV lanes, but the CAVs and HVs all have a right of way on regular links. The paths for CAV and HV will also be different after implementing the CAV lanes. There will lead to the different path choice for CAVs and HVs. For instance, in Figure 1, there are two paths for HVs for OD pairs $(1,3)$, that is, link 2 and link $1 \rightarrow \operatorname{link} 3$, but CAVs have two more path options, namely, link $2^{\prime}$ and link $1 \rightarrow$ link $3^{\prime}$. 


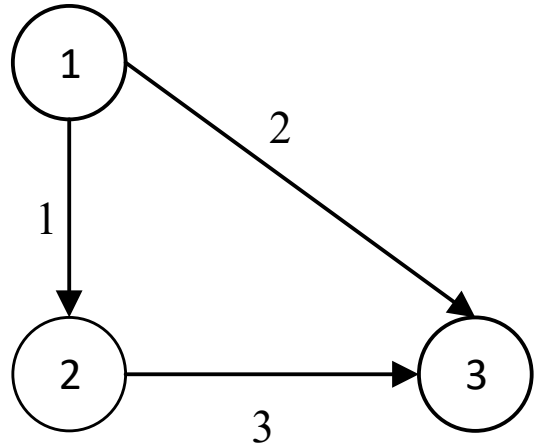

(a) Original network topology

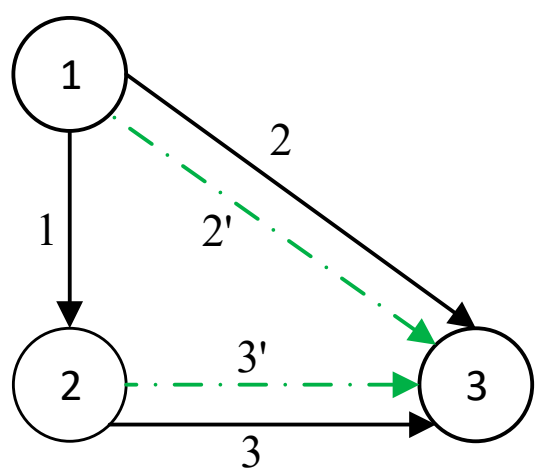

(b) Revised network topology

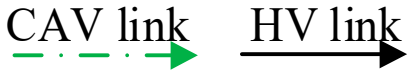

Figure 1. A simple network example: (a) original network topology; and (b) revised network topology.

Benefiting from advanced vehicle automation technology, infrastructure equipment, vehicle-to-vehicle communication, CAVs could travel with a short headway on the dedicated CAV lanes, which makes CAV lanes have a higher capacity [19]. According to existing research, the capacity of dedicated CAV lanes is 2-3 times that of regular lanes [39]. On the other hand, we assume the regular link capacity is not improved, because CAVs are affected by the HVs and fail to travel with a short headway.

\subsection{Optimization Objectives}

\subsubsection{Vehicle Emission Cost}

It is known that the main components in vehicle emissions are carbon monoxide (CO), volatile organic compounds (VOC), and nitrogen oxides (NOx). In this paper, the TRANSYT-7F average-speed emission estimation model developed by Penic and Upchurch [40], i.e., Equation (2), was selected to calculate the vehicle emission. The network total emission cost was regarded as an evaluation indicator for the sustainable dedicated CAV lanes design problem, as follows:

$$
Z_{E}=\sum_{a \in A} \sum_{m \in M} e_{a}^{m} x_{a}^{m} \cdot \forall a \in A, m \in M
$$

where $x_{a}^{m}$ is the traffic volume of link $a . e_{a}^{m}$ represents the emission of vehicle type $m$ from link $a$, which can be written as

$$
e_{a}^{m}=\sum_{p} \phi^{p} \frac{\mathcal{A}^{p} \exp \left(\mathcal{B}^{p_{v_{a}}}\right) L_{a}}{\mathcal{C}^{p} v_{a}} \rho^{m} \quad \forall a \in A, m \in M
$$

where $L_{a}$ is the length of link $a ; \rho^{m}$ is the emission factor for vehicle type $m . p$ is the index of pollutants including $\mathrm{CO}, \mathrm{NOx}$, and VOC. $\mathcal{A}^{p}, \mathcal{B}^{p}$, and $\mathcal{C}^{p}$ are the model parameters. $\phi^{p}$ is the coefficients of pollutant $p . v_{a}$ denotes the average travel speed on link $a$, which depends on the travel time $t_{a}^{m} . t_{a}^{m}$ is computed using the Bureau of Public Roads function [41], as follows:

$$
\begin{gathered}
t_{a}^{m}=t_{a}^{0}\left[1+\alpha\left(\frac{x_{a}^{m}}{\Lambda_{a}^{m}}\right)^{\beta}\right] \quad \forall a \in A, m \in M \\
x_{a}^{m}=\sum_{w \in W} \sum_{r \in R} \sum_{m \in M} \delta_{a}^{r, w} f_{r, w}^{m} \quad \forall a \in A, m \in M, r \in R
\end{gathered}
$$

where $t_{a}^{0}$ denotes the free-flow travel time; $\alpha, \beta$ are the parameters; $f_{r, w}^{m}$ indicates the traffic flow of vehicle type $m$ on path $r$ for OD $w . \delta_{a}^{r, w}$ is the $0-1$ link-path incidence indicator. $\Lambda_{a}^{m}$ 
is the capacity of link $a$, which depends on the link type. In detail, the formulation of $\Lambda_{a}^{m}$ can be written as

$$
\begin{gathered}
\Lambda_{a}^{1}=\mathbb{C} \sum_{i \in k} y_{k}^{i} \quad \forall a \in A, i \in k, k \in K \\
\Lambda_{a}^{2}=\mathbb{C}\left(Y_{k}-\sum_{i \in k} y_{k}^{i}\right) \forall a \in A, i \in k, k \in K \\
y_{k}^{i}=\{0,1\} \quad \forall i \in k, k \in K
\end{gathered}
$$

where $\hat{\mathbb{C}}$ and $\mathbb{C}$ denote the capacity of one $\mathrm{CAV}$ lane and regular lane, respectively. $y_{k}^{i}$ is a binary decision variable. If $y_{k}^{i}=1$, this means the CAV lane $i$ is deployed on link pair $k$, and 0 otherwise. $Y_{k}$ represents the total number of lanes for link pair $k$.

\subsubsection{CAV Lanes Construction Cost}

Optimizing the construction cost of deploying dedicated CAV lanes is important because of the limited available budget. Decision-makers aim to obtain the optimal implement scheme under a limited budget. The total construction cost of implementing CAV lanes in a network is calculated as

$$
Z_{C}=\sum_{k \in K} \sum_{i \in k} y_{k}^{i} l_{i} u_{i} \forall a \in A, i \in k, k \in K
$$

where $l_{i}$ is the length of the lane $i$; and $u_{i}$ is the unit cost of CAV lane construction.

\subsubsection{System Travel Time Cost}

The total travel time cost is the sum of all vehicle time costs on the road network, as follows:

$$
Z_{T}=\sum_{m \in M} \sum_{a \in A} t_{a}^{m} x_{a}^{m} \gamma^{m} \forall a \in A, m \in M
$$

where $\gamma^{m}$ is the value of time (VOT) for the vehicle type $m ; x_{a}^{m}$ denotes the equilibrium traffic flow.

\subsection{Multi-Class Network Equilibrium}

In this work, we assumed that the travel choice follows Wardrop's user equilibrium (UE) principle [42]. The travel time on a path $c_{r, w}^{m}$ is the sum of travel time spent on all links used by the path, as follows

$$
c_{r, w}^{m}=\sum_{a \in A} \delta_{a}^{r, w} t_{a}^{m} \quad \forall a \in A
$$

The multi-class network equilibrium condition can be formulated as [43]

$$
\begin{gathered}
f_{r, w}^{m}\left(c_{r, w}^{m}-c_{w}^{m}\right)=0 \quad \forall m \in M, r \in R, w \in W \\
c_{r, w}^{m}-c_{w}^{m} \geq 0 \quad \forall m \in M, r \in R, w \in W \\
\sum_{r \in R_{w}^{m}} f_{r, w}^{m}=q_{w}^{m} \quad \forall m \in M, r \in R_{w}^{m}, w \in W \\
f_{r, w}^{m} \geq 0 \quad \forall m \in M, r \in R
\end{gathered}
$$

where $c_{w}^{m}$ is the minimum travel time by vehicle type $m$ in OD pair $w$. Equations (11) and (12) indicate the travel mode $m$ will use path $r$ only if the travel time of the path equal to the minimum travel time of that OD pair. Constraints (13) and (14) are OD demand conservation and non-negativity constraints. 


\section{Mathematical Model}

In this section, the dedicated CAV lanes optimal implementation problem was formulated as a bi-level programming model. The upper-level model aims to determine the optimal location to deploy CAV lanes. The lower-level model is expressed as the multi-class network equilibrium problem, and is expressed by a complementarity formulation. As a result, the multi-objective bi-level model can be formulated as follows:

$$
\min _{y}\left(\begin{array}{c}
Z_{T}(y, x(y)) \\
Z_{E}(y, x(y)) \\
Z_{C}(y)
\end{array}\right)
$$

subject to:

$$
\begin{gathered}
\sum_{k \in K} \sum_{i \in k} y_{k}^{i} l_{i} u_{i} \leq B \quad \forall k \in K, r \in R, w \in W \\
\Lambda_{a}^{1}=\hat{\mathbb{C}} \sum_{i \in k} y_{k}^{i} \quad \forall i \in k, k \in K \\
\Lambda_{a}^{2}=\mathbb{C}\left(Y_{k}-\sum_{i \in k} y_{k}^{i}\right) \forall i \in k, k \in K \\
y_{k}^{i}=\{0,1\} \quad \forall i \in k, k \in K \\
c_{r, w}^{m}=\sum_{a \in A} \delta_{a}^{r, w} t_{a}^{m} \quad \forall r \in R, m \in M, a \in A \\
f_{r, w}^{m}\left(c_{r, w}^{m}-c_{w}^{m}\right)=0 \quad \forall m \in M, r \in R, w \in W \\
c_{r, w}^{m}-c_{w}^{m} \geq 0 \quad \forall m \in M, r \in R, w \in W \\
\sum_{r \in R_{w}^{m}} f_{r, w}^{m}=q_{w}^{m} \quad \forall m \in M, r \in R_{w}^{m}, w \in W \\
f_{r, w}^{m} \geq 0 \quad \forall m \in M, r \in R
\end{gathered}
$$

where $y$ represents the decision variable, which is the vector of the CAV lanes' implementation. $x(y)$ is the link equilibrium flow based on $y$. Constraint (16) is the budget restriction, in which $B$ denotes the budget of CAV lanes implement. Constraints (17)-(18) denote that the link capacity equals the sum of the capacity of lanes. Constraint (19) is the binary variable constraint. Constraint (20) represents the link accumulation flow. Constraints (21)-(24) indicate the multi-class network equilibrium conditions.

\section{Solution Algorithm}

In this section, two algorithms are introduced to solve the dedicated CAV lanes optimal implement problem. The upper-level model was to determine the optimal implementation scheme by minimizing the objective value, which is difficult to handle because it belongs to an NP (non-deterministic polynomial) hard problem. Heuristic algorithms are generally regarded as effective methods to solve NP hard problems. Hence, in this paper, the nondominated sorting genetic algorithm II (NSGA-II) [44] was adopted to solve the upper-level model, which has multiple objectives. The low-level problem is the multi-class network equilibrium, which can be solved by the diagonalized Frank-Wolfe (DFW) algorithm [45]. Figure 2 draws the overall framework of the solution approach.

NSGA-II, introduced by Deb et al., is an effective algorithm to deal with multi-objective optimization problems, which is capable of efficiently constructing the Pareto front by three operators: (1) non-dominant solution sorting operator; (2) individually crowded distance operator; and (3) elite strategy selection operator. Figure 3 shows the detailed flowchart of NSGA-II. 


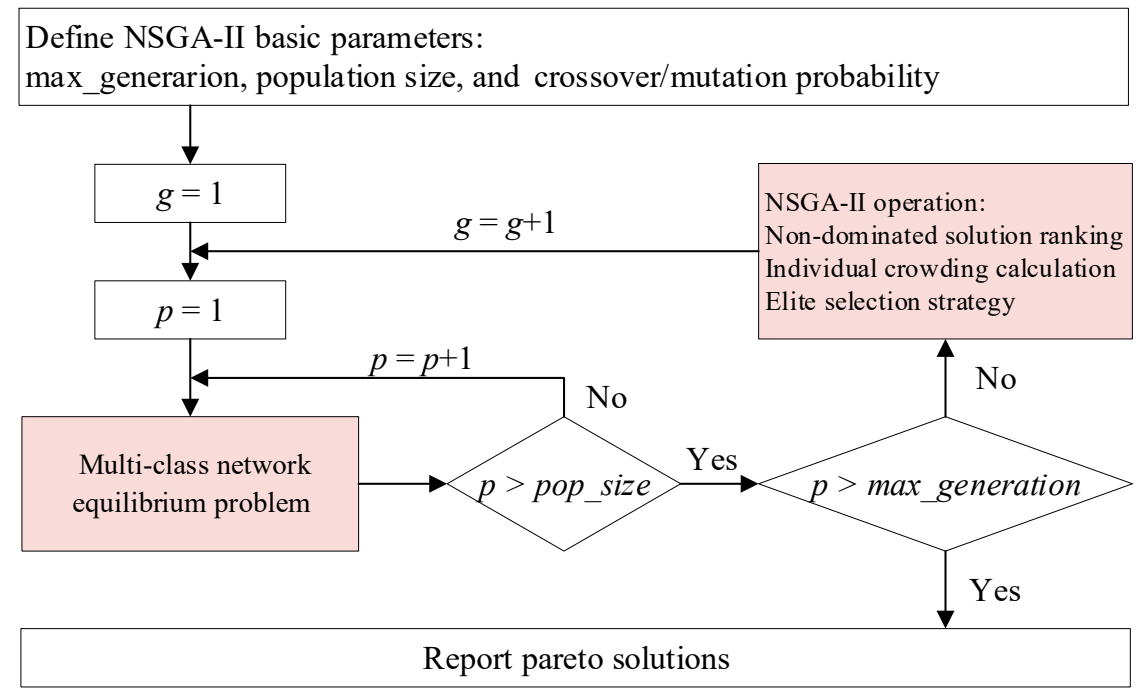

Figure 2. The overall framework of the solution approach.

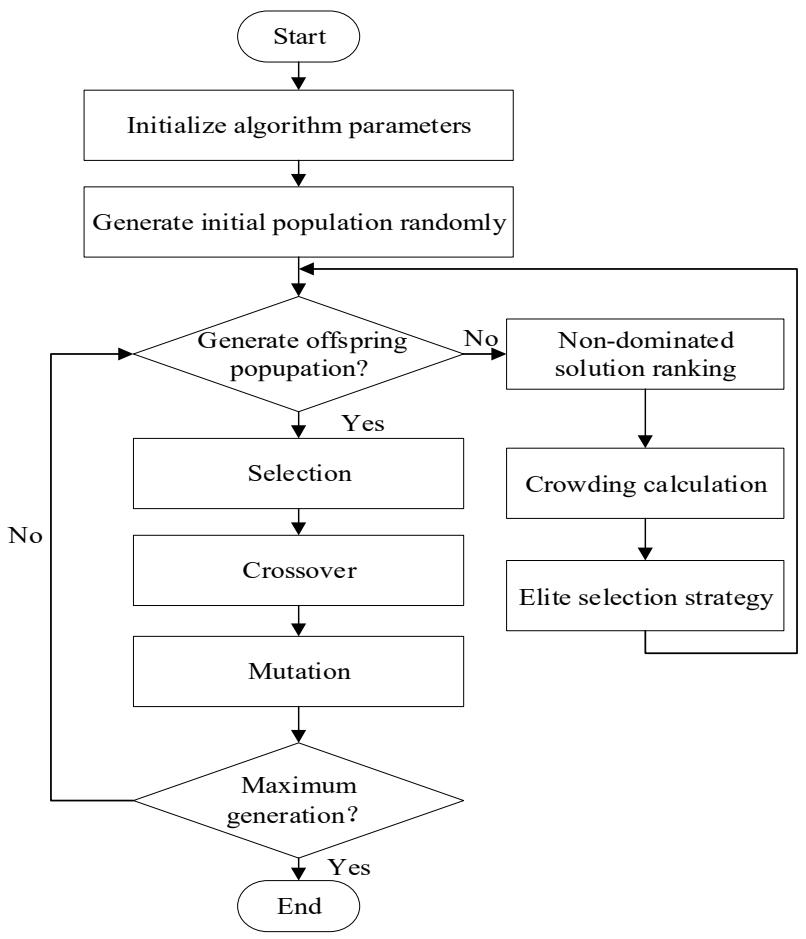

Figure 3. The flowchart of NSGA-II.

Due to the asymmetric impact on link cost between travel modes, it is difficult to solve the heterogeneous traffic streams equilibrium problems directly. The diagonalization algorithm as an efficient method usually is adapted to handle the heterogeneous traffic streams equilibrium problems. The diagonalization algorithm was first proposed by Abdulaal and LeBlanc [46] to solve the traffic assignment problem, and then Sheffi [47] implemented it for the traffic equilibrium problem with asymmetric link interactions.

Note that the diagonalization algorithm is usually combined with a single-class traffic assignment algorithm to solve mixed traffic assignment problems. Specifically, the FrankWolfe algorithm is adopted in this work, i.e., a diagonalized Frank-Wolfe (DFW) algorithm is used to deal with the multi-class network equilibrium problems. The detailed steps of DFW are shown in Algorithm 1. 


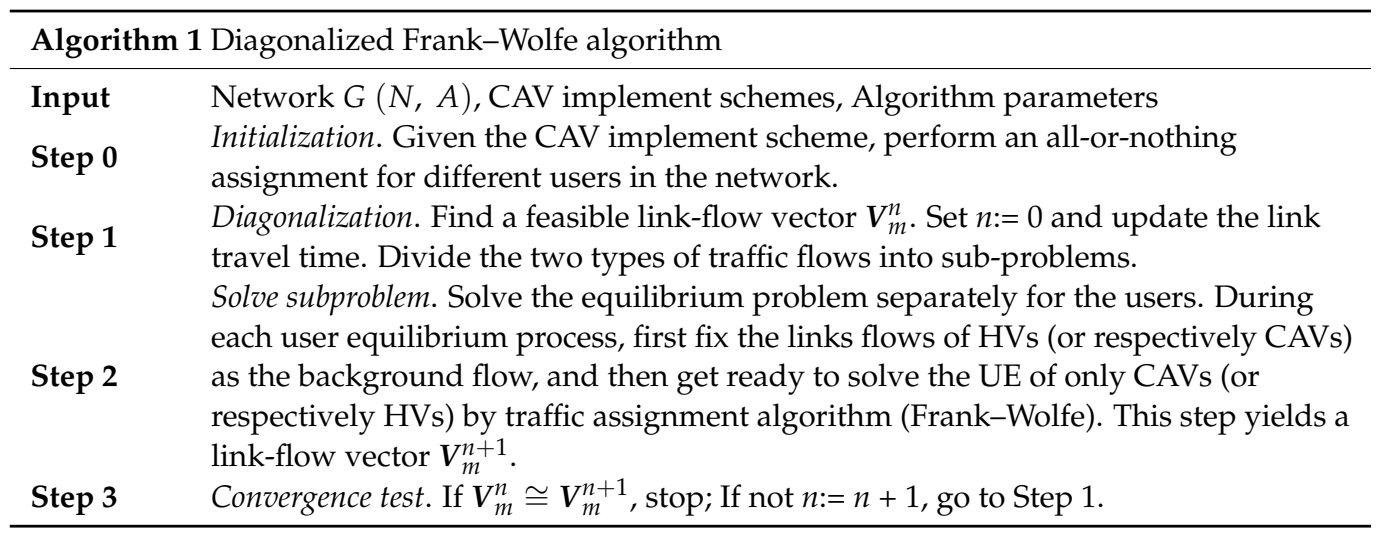

The sub-problem mentioned in Algorithm 1 refers to the UE problem. In other words, the solution of the sub-problem is equal to the solution of user equilibrium. The UE solution can be obtained by solving following Beckmann's transformation:

$$
\min _{\omega} \sum_{a \in A} \int_{0}^{x_{a}} t_{a}(\omega) d_{\omega}
$$

Subject to:

$$
\begin{gathered}
x_{a}^{m}=\sum_{w \in W} \sum_{r \in R} \sum_{m \in M} \delta_{a}^{r, w} f_{r, w}^{m} \\
\sum_{r \in R_{w}^{m}} f_{r, w}^{m}=q_{w}^{m} \\
f_{r, w}^{m} \geq 0
\end{gathered}
$$

\section{Numerical Examples}

\subsection{Nguyen-Dupuis Network}

The proposed model and algorithm are first implemented in the Nguyen-Dupuis network (Figure 4), which consists of 13 nodes, 19 links, and four OD pairs. The total demand for OD pairs $(1,12),(1,13),(3,12)$, and $(3,13)$ is $8,8,6$, and $6\left(10^{3}\right.$ vehicles $)$, respectively. The penetration rate of CAVs is assumed to be $50 \%$ and identical for all OD pairs. The parameters in travel time function parameters are $\alpha=0.15$, and $\beta=4$. The detailed network attributes are listed in Table 1 , including the length $\left(L_{a}\right)$, free-flow travel time $\left(t_{a}^{0}\right)$, and initial capacity $\left(\Lambda_{a}^{0}\right)$. Table 2 shows the detailed parameters and coefficients of three pollutants.

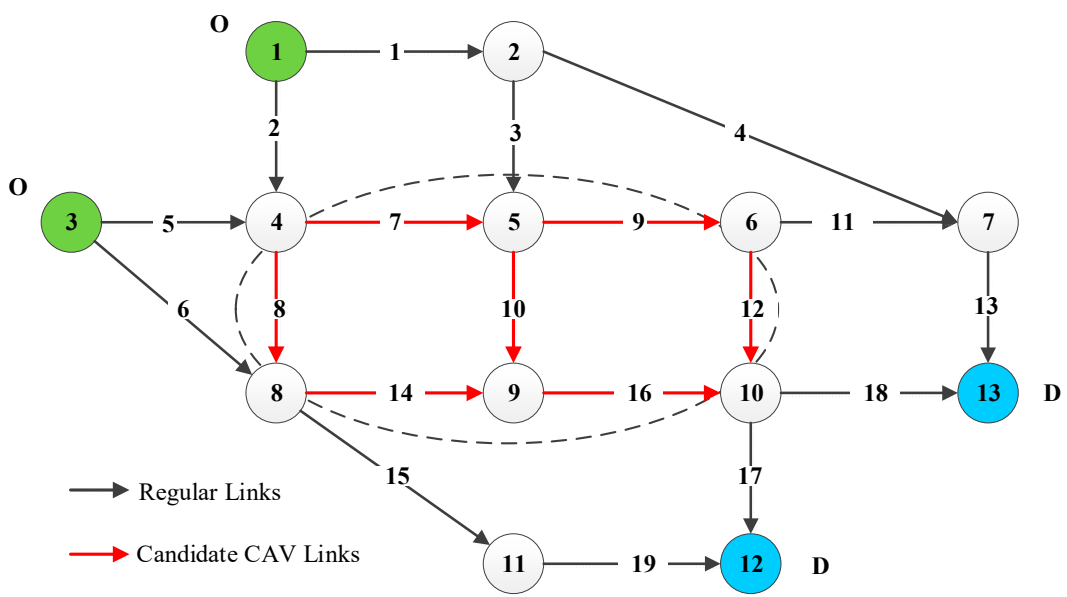

Figure 4. The Nguyen-Dupuis network. 
Table 1. Link attributes of modified Nguyen-Dupius network.

\begin{tabular}{cccccccccc}
\hline Link & $\boldsymbol{t}_{\boldsymbol{a}}^{0}$ & \#Lanes & Capacity & Length & Link & $\boldsymbol{t}_{\boldsymbol{a}}^{0}$ & \#Lanes & Capacity & Length \\
\hline 1 & 9 & 3 & 3000 & 5.4 & 11 & 2 & 3 & 3000 & 3 \\
2 & 7 & 4 & 4000 & 4.2 & 12 & 9 & 4 & 4000 & 5.4 \\
3 & 7 & 3 & 3000 & 4.2 & 13 & 9 & 3 & 3000 & 5.4 \\
4 & 14 & 2 & 2000 & 8.4 & 14 & 10 & 4 & 4000 & 6 \\
5 & 9 & 3 & 3000 & 5.4 & 15 & 9 & 2 & 2000 & 5.4 \\
6 & 12 & 3 & 3000 & 7.2 & 16 & 6 & 4 & 4000 & 3.6 \\
7 & 3 & 4 & 4000 & 1.8 & 17 & 5 & 3 & 3000 & 3 \\
8 & 9 & 3 & 3000 & 5.4 & 18 & 9 & 4 & 4000 & 5.4 \\
9 & 5 & 3 & 3000 & 3 & 19 & 11 & 3 & 3000 & 6.6 \\
10 & 13 & 3 & 3000 & 7.8 & & & & & \\
\hline
\end{tabular}

Table 2. Parameters and coefficients of three pollutants.

\begin{tabular}{cccc}
\hline Parameters & Pollutant & & \\
\hline & CO & VOC & NOx \\
\hline $\mathcal{A}^{p}(\mathrm{~g} / \mathrm{ft}$. per veh $)$ & 3.3963 & 2.7843 & 1.5718 \\
$\mathcal{B}^{p}(\mathrm{~s} / \mathrm{ft})$ & 0.014561 & 0.015062 & 0.040732 \\
$\mathcal{C}^{p}(\mathrm{~s} / \mathrm{ft})$ & 1000 & 10000 & 10000 \\
$\phi^{p}(\$ / \mathrm{g})$ & 0.00051 & 0.00136 & 0.00103 \\
\hline
\end{tabular}

\subsubsection{The Base Scenario}

In the base scenario, we compared the three scenarios to investigate the benefit of implementing dedicated CAV lanes. The first scenario is the basic network without CAV lanes implementation and CAVs. The second scenario was the network with CAVs but without CAV lanes. The third scenario is the modified network with CAVs and CAV lanes implementation. Table 3 and Figure 5 show detailed results for the three scenarios. Given that there is more than one Pareto optimal solution, we take the mean of Pareto fronts as the optimal solution to make a comparison. From Table 3, we know that implementing CAV lanes in the road network cannot only reduce system travel time cost, but also reduce the vehicle emission cost. More specifically, compared to scenario 1, scenario 2 could reduce the total emission costs by $9.9 \%$, which indicates the emergence of CAV benefits to reduce emission costs. Compared with scenarios 1 and 2, scenario 3 contributes to reducing the total travel time cost by $12.03 \%$. Similarly, scenario 3 reduces the total emission cost by $19 \%$ and $10.42 \%$, compared to scenarios 1 and 2, respectively. As a result, implementing dedicated CAV lanes could further decrease emission costs and improve traffic efficiency. The adoption of CAVs and implementing dedicated CAV lanes will be conducive to sustainable transportation development while ensuring travel efficiency.

Figure 6a presents the changes of three pollutants in the three scenarios. Specifically, compared to scenario 1 and scenario 2, CO emissions decrease by $19.5 \%$ and $10.5 \%$, respectively, in scenario 3. Similarly, NOx emissions would reduce by $17.5 \%$ and $7 \%$; and the VOC emissions reduce by $19.4 \%$ and $10.3 \%$. Obviously, implementing dedicated CAV lanes will reduce pollutant emissions, which is reasonable. An explanation is that the dedicated CAV lanes could promote traffic efficiency by increasing link capacity. On the other hand, advance connected autonomous driving and communication technologies of CAVs are beneficial to further reduce energy consumption. Figure $6 \mathrm{~b}$ shows the result of energy consumption and $\mathrm{CO}_{2}$ emission for three scenarios. More specifically, the total energy consumption in scenario 3 decreases by $19.5 \%$ and $9.4 \%$ compared to scenarios 1 and 2 . Similarly, total $\mathrm{CO}_{2}$ emissions could reduce by $0.4 \%$ and $9.5 \%$. The results support that CAVs and the deployment of CAV lanes could reduce the energy consumption and $\mathrm{CO} 2$ emission. In other words, the adoption of CAVs will reduce the negative effects of road transportation on the environment. 
Table 3. Comparison results in the three scenarios in the Nguyen-Dupuis network.

\begin{tabular}{|c|c|c|c|}
\hline & Total Travel Time Cost (USD $10^{5}$ ) & Total Emission Cost (USD) & Total Construction Cost (USD 106) \\
\hline Scenario 1 & 8.53 & 660.72 & - \\
\hline Scenario 2 & 8.53 & 595.09 & - \\
\hline Scenario 3 & 7.53 & 535.20 & 1.62 \\
\hline
\end{tabular}

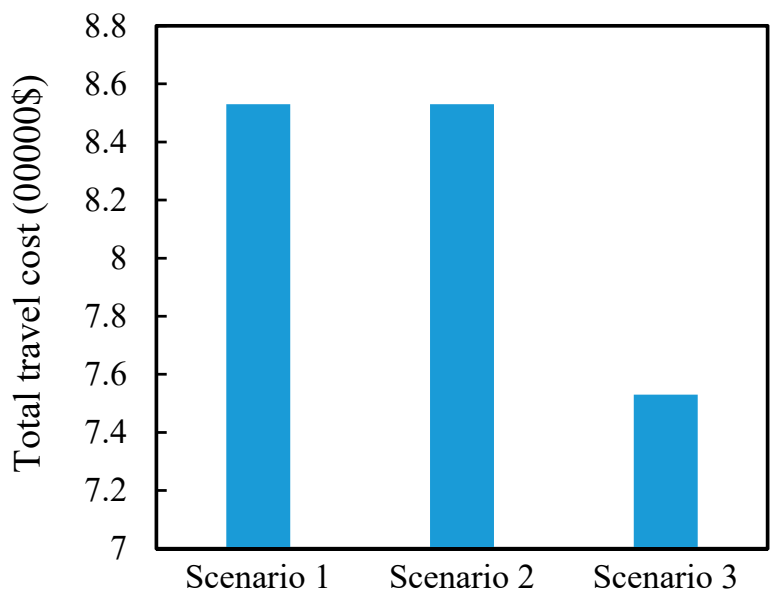

(a)

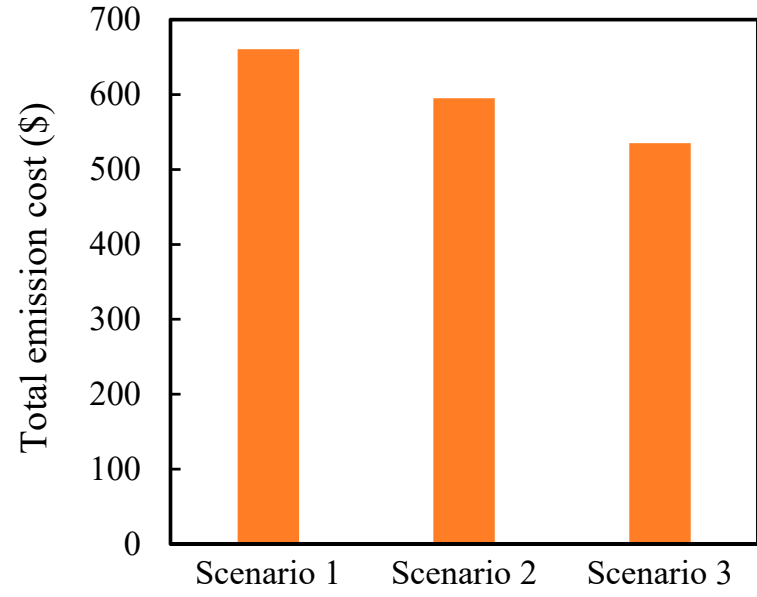

(b)

Figure 5. Total travel cost (a) and total emission cost (b) in the Nguyen-Dupuis network.

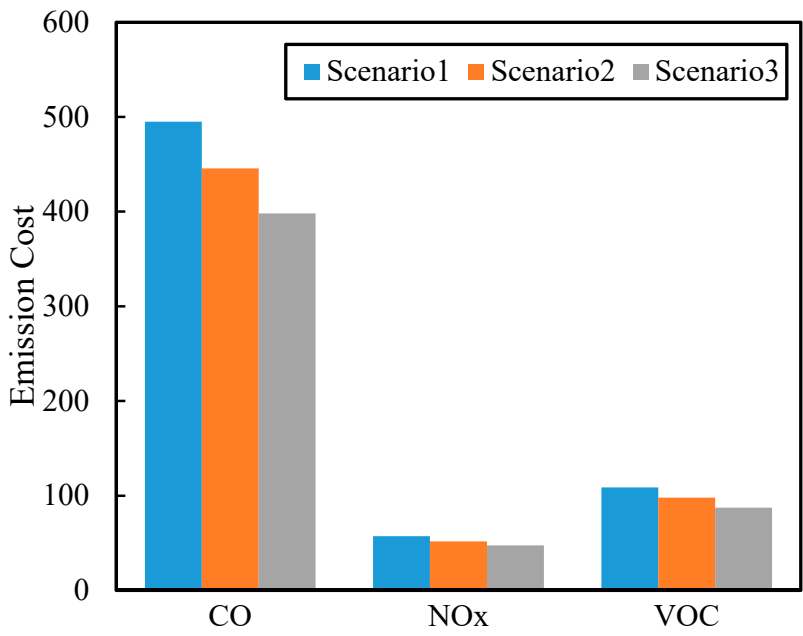

(a)

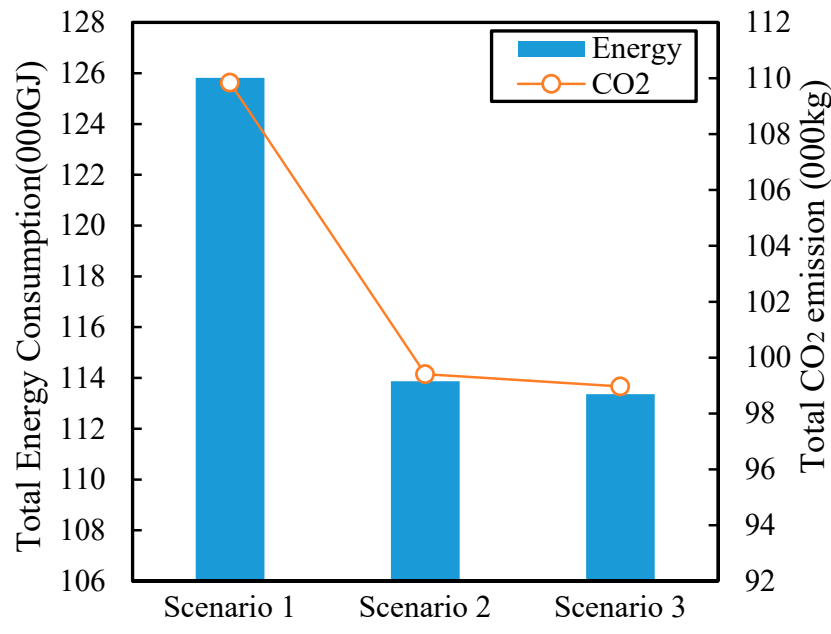

(b)

Figure 6. Emission cost (a) and total energy consumption (b) in the Nguyen-Dupuis network.

Figure 7 presents the Pareto front of the dedicated CAV lanes implementation schemes and the relationship between the two objectives in the Nguyen-Dupuis network. The result shows that there are evident trade-offs between minimizing the emission cost and CAV lanes construction cost, as well as the minimizations of the travel cost and construction cost. In contrast, emission cost and construction cost show consistent trends to some extent. Moreover, many potential benefits can be obtained from implementing dedicated CAV lanes, which will greatly reduce the total travel time and emission before the construction cost up to $2 \times 10^{6} \$$. After this value, when the construction cost varies from $2 \times 10^{6} \$$ to $3.5 \times 10^{6} \$$, the total travel time cost and emission cost are relatively stable. The benefits from CAV lanes implementation for network performance are very weak. 


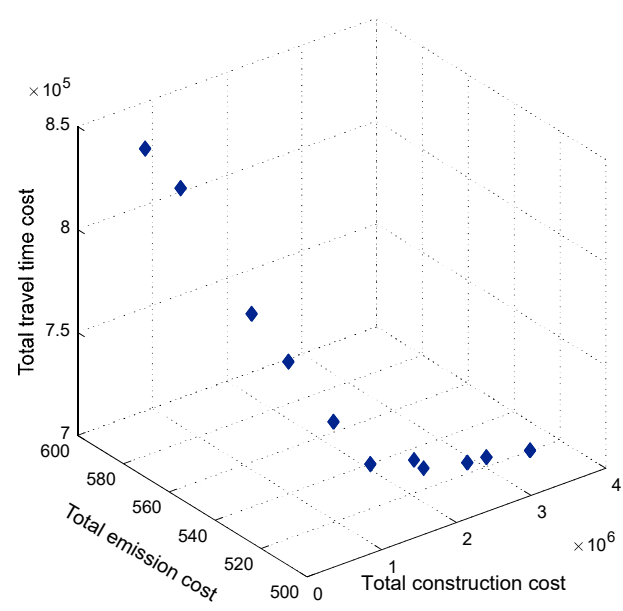

(a)

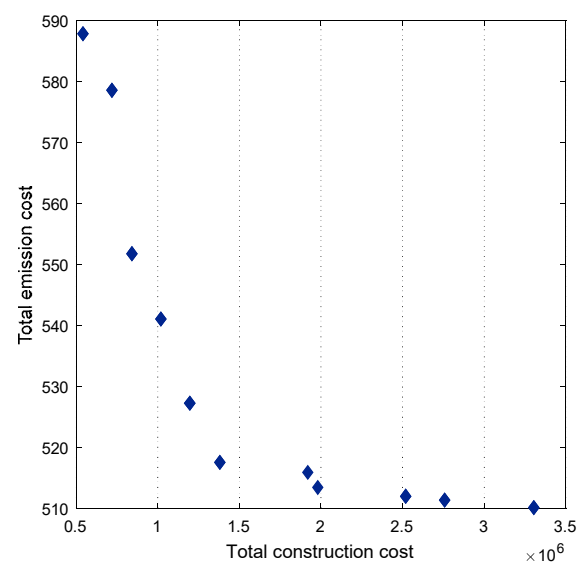

(c)

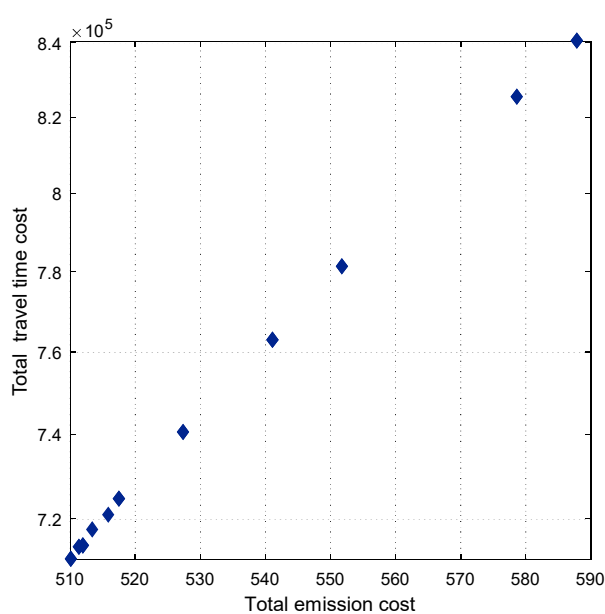

(b)

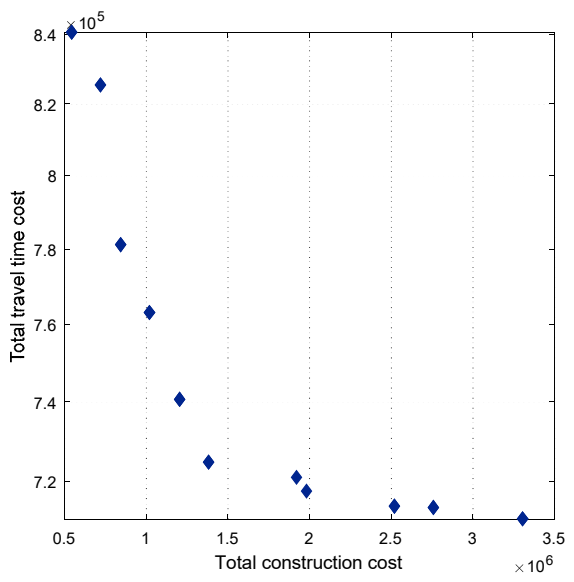

(d)

Figure 7. Pareto fronts of the Nguyen-Dupuis network (a) and the relation between the three goals in pairs $(\mathbf{b}-\mathbf{d})$.

Based on the above analysis, we concluded that performing multi-objective dedicated CAV lanes network design is crucial. In general, enough Pareto optimal solutions are acquired, which offers the decision-makers considerable alternative solutions. Sometimes, decision-makers need to make choices between investment budget and transportation environment. A detailed analysis of the relations between the three objectives can provide useful information for environmentally sustainable transportation planning.

\subsubsection{Sensitivity Analyses}

To further explore the system performance under the different model parameters, sensitivity analyses were performed in this section, in terms of CAV market penetration, CAV lane capacity, and vehicle travel speed. It is worth noting that the sensitivity analysis is based on the dedicated CAV implement schemes obtained from the base scenario. The corresponding CAV implementation scheme is shown in Figure 8, one of the Pareto fronts closest to the mean solution. In Figure 8, we know that link $4 \rightarrow 5$, link $5 \rightarrow 6$, and link $6 \rightarrow 10$ are selected to deploy dedicated CAV lanes. 


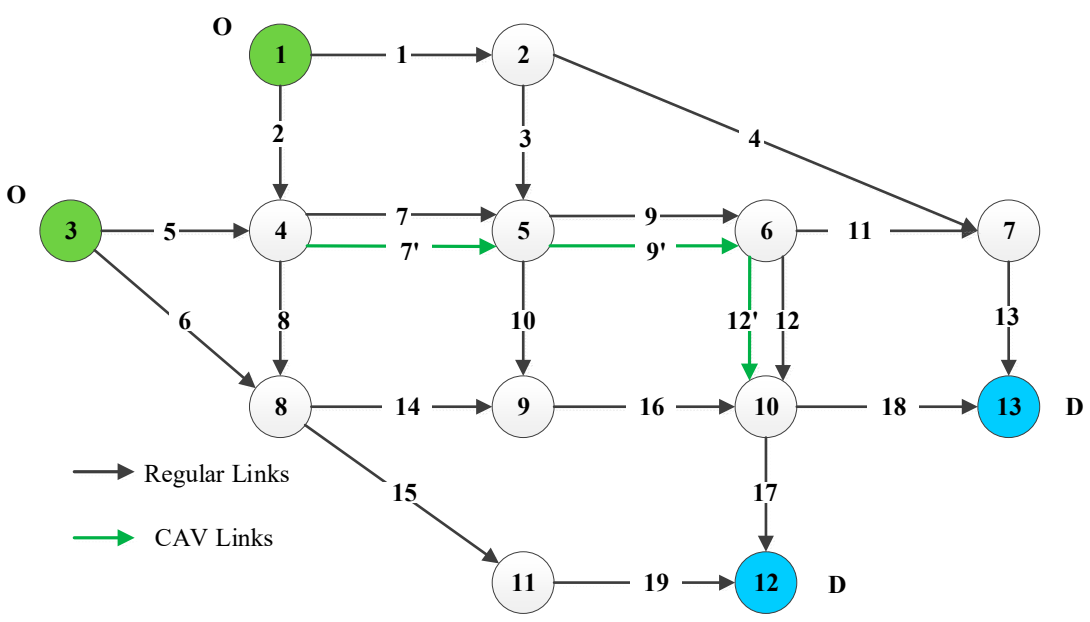

Figure 8. The specific CAV implement scheme.

(1) CAV market penetration

Figure 9 presents the effect of CAV market penetration on total travel cost and total emission cost for two scenarios. The CAV market penetration varies from $10 \%$ to $90 \%$. With different CAV market penetration, implementing dedicated CAV lanes could reduce the total travel cost and emission cost, except when CAV market penetration is $10 \%$. An explanation is that implementing dedicated CAV lanes on a network with a lower market share will cause the waste of road resources, which brings adverse effects. In other words, the number of CAV lanes should match the CAV market share to reach an equilibrium state. This is also confirmed in the corresponding Figure 9a, where the total travel time will decrease as the penetration rate increases before the CAV penetration rate reaches $50 \%$. After more than 50\%, the total travel time remains relatively stable (Figure 9a). This is because the optimal dedicated CAV lanes solution is obtained from the base scenario, in which the CAV penetration is $50 \%$. The total travel cost remains stable after the CAV market penetration is over $50 \%$ because the dedicated CAV lanes are fully utilized. In addition, Figure 10 shows the change of three pollutants in the two scenarios.

(2) CAV lane capacity

Figure 11 denotes the effect of CAV lane capacity on the total travel cost and total emission cost considering two situations, i.e., the capacity of a CAV lane is two and three times the capacity of a regular lane. As shown in Figure 11a, a larger capacity for dedicated CAV lanes will reduce the system's total travel cost. This conclusion can also be confirmed by the Pareto relationship between the total travel cost and CAV construction cost as shown in Figure 7d. Figure 11b further affirms that greater CAV lane capacity reduces the total emission cost. In addition, Figure $7 \mathrm{~d}$ shows that a larger $\mathrm{CAV}$ market penetration decreases the total emission cost. Specifically, compared to the two-time capacity increase, the three-time capacity increase would reduce the total travel cost and total emission cost by $5.5 \%$ and $4.6 \%$, respectively, when the CAV market penetration is $50 \%$. Intuitively, Figure 12 reports the change of three pollutants (CO, NOx, and VOC) under the two CAV lane capacity situations.

(3) Vehicle travel speed

The above analysis is based on the consistent relationship between minimizing the total travel time cost and total emission cost. However, the relation between minimizing the total travel time cost and total emission cost is not necessarily always consistent in terms of high vehicle speed $[34,48]$. For example, Figure 13 shows the Pareto fronts and the relationship considering the high vehicle speed. Base on Figure 13, we found the conflict relation between the total travel time and total emission cost when the total travel time cost is less than $3.45 \times 10^{5}$. However, the Pareto relation between the total travel time cost and construction cost is consistent. 


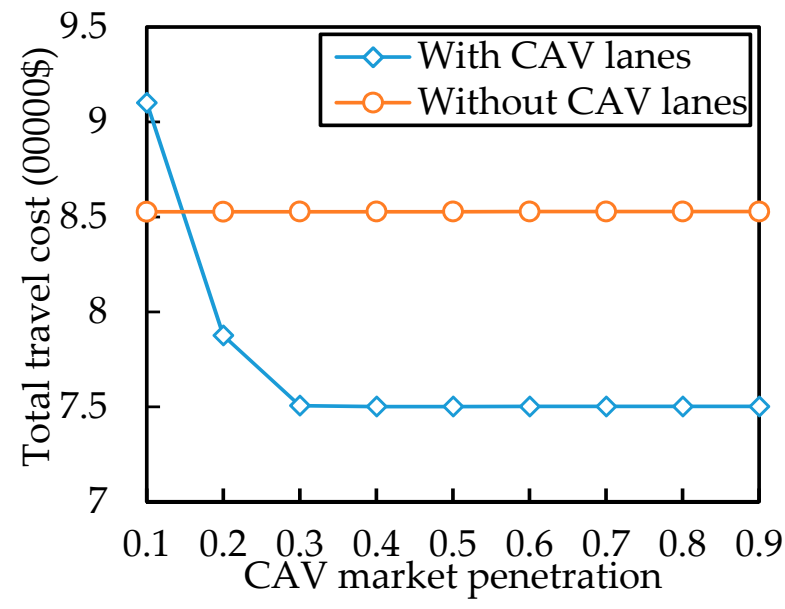

(a)

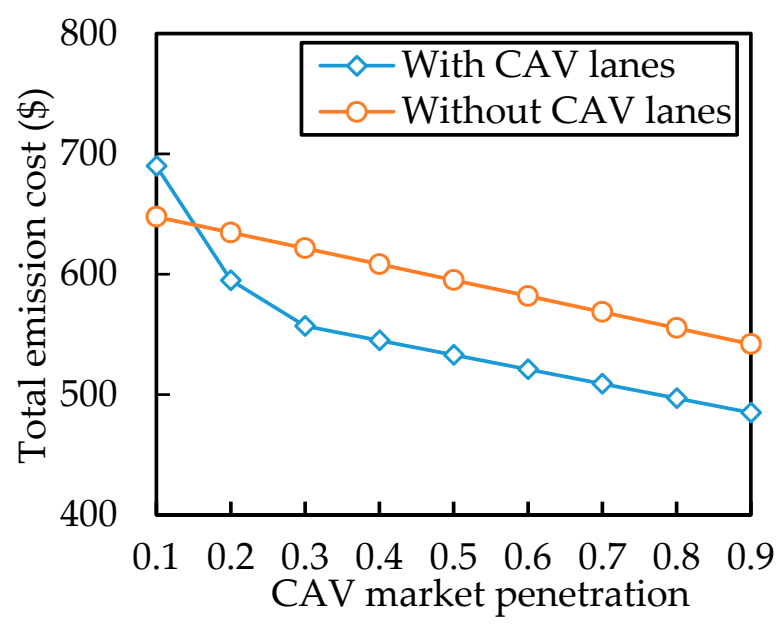

(b)

Figure 9. Effect of CAV market penetration on the total travel cost (a) and total emission cost (b).

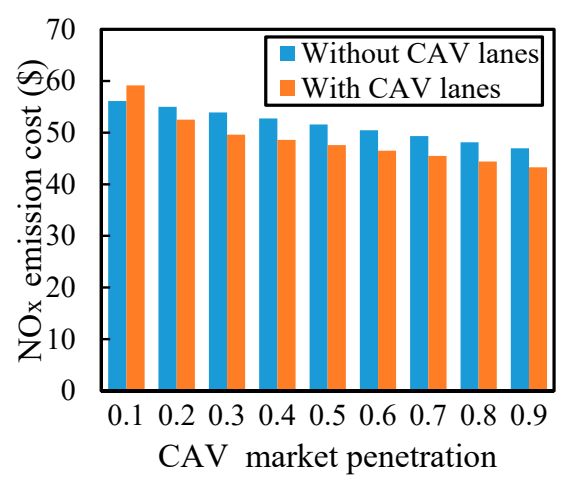

(a)

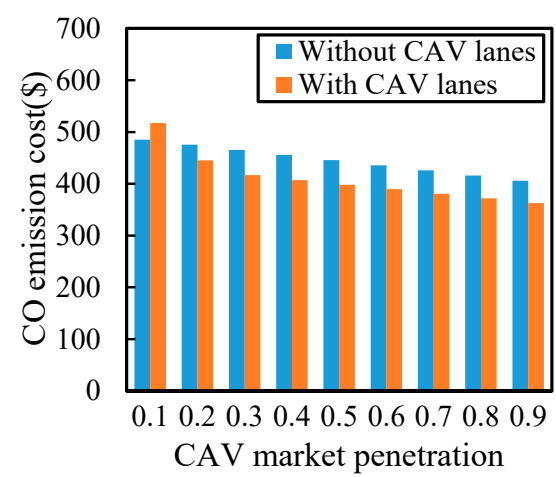

(b)

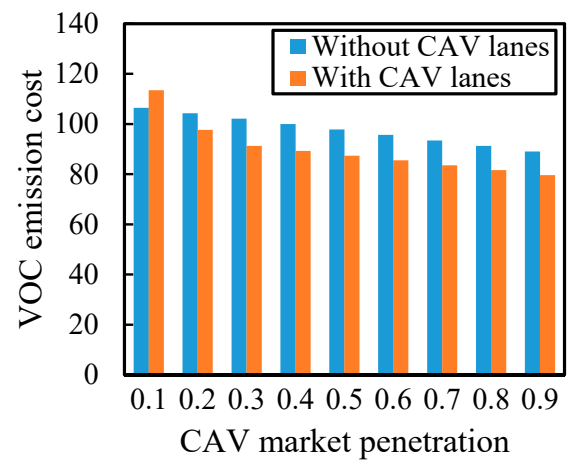

(c)

Figure 10. Effect of CAV market penetration on three pollutants in two scenarios: NOx emission cost (a); CO emission cost (b); and VOC emission cost (c).

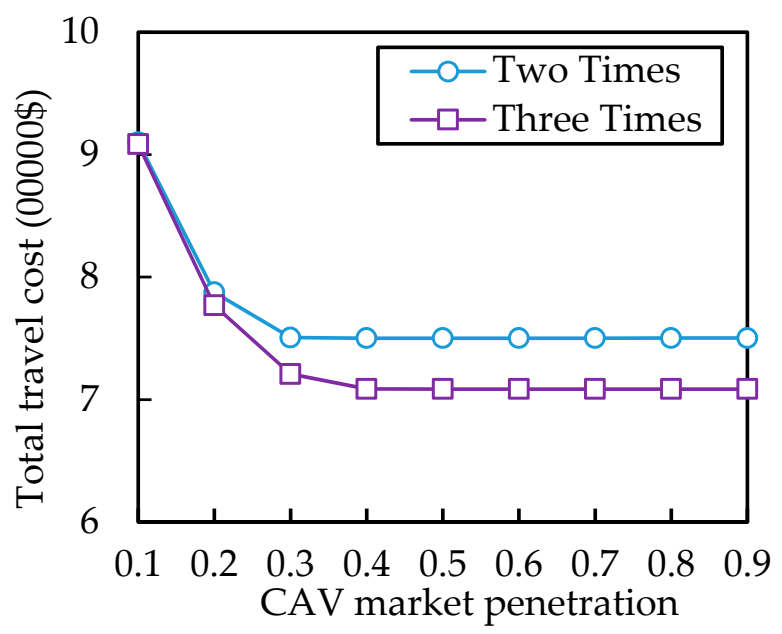

(a)

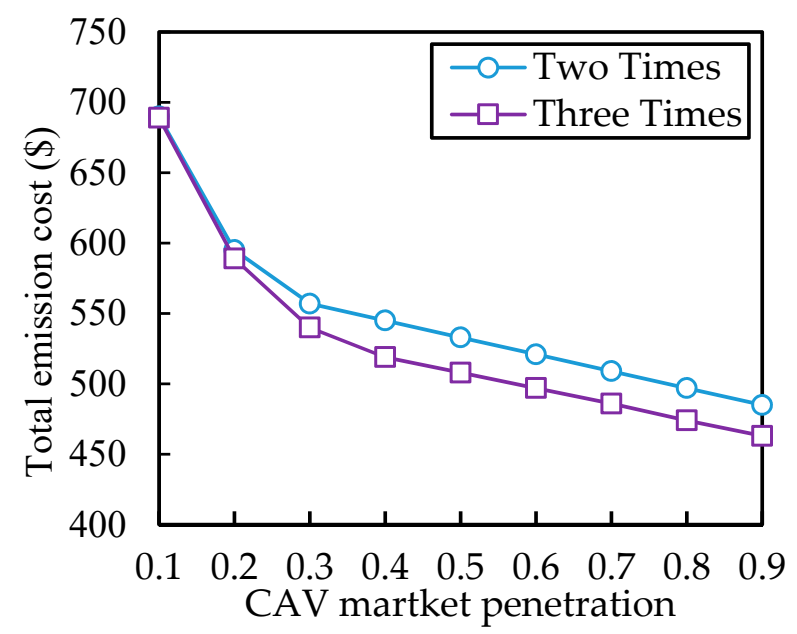

(b)

Figure 11. Effect of the capacity of the CAV lane on the total travel cost (a) and the total emission cost (b). 


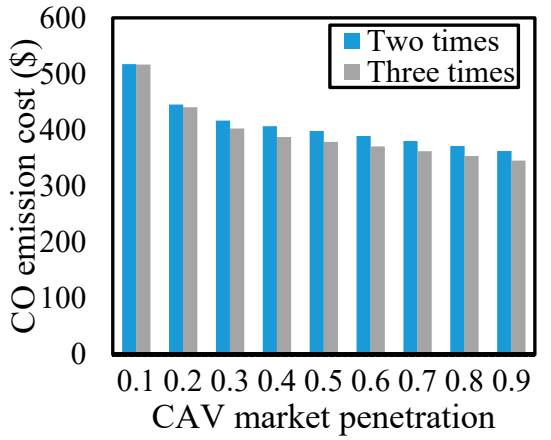

(a)

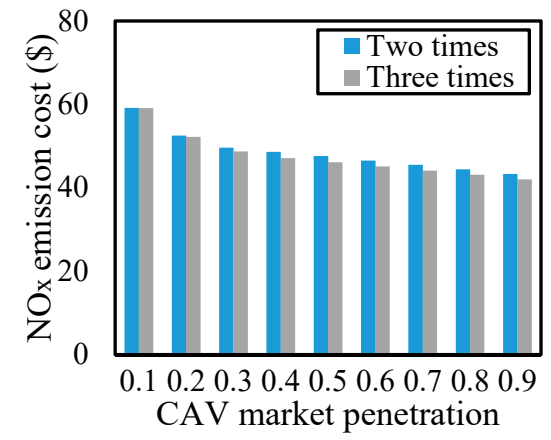

(b)

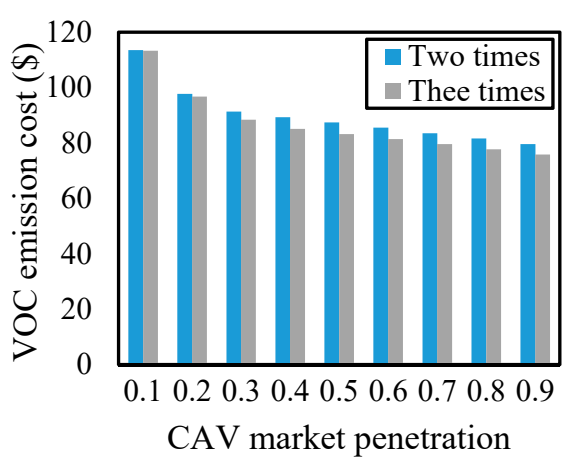

(c)

Figure 12. Effect of CAV market penetration on three pollutants for two capacity scenarios: CO emission cost (a); NOx emission cost (b); and VOC emission cost (c).

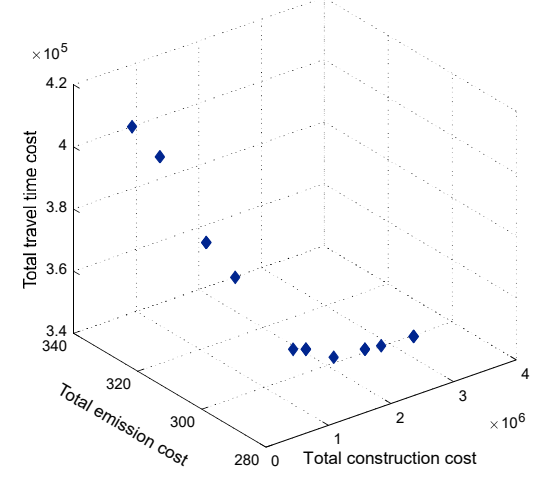

(a)

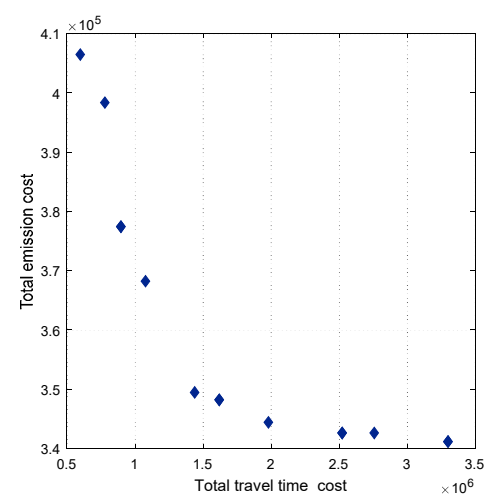

(c)

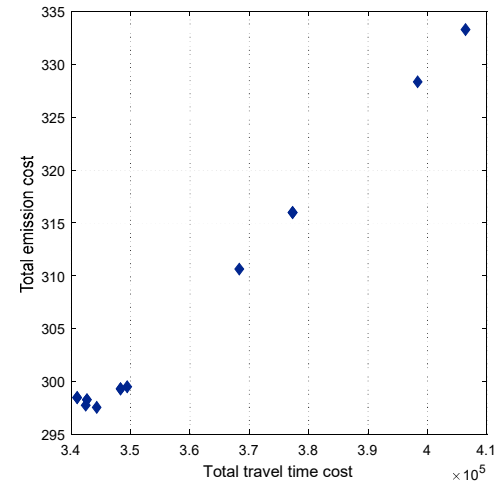

(b)

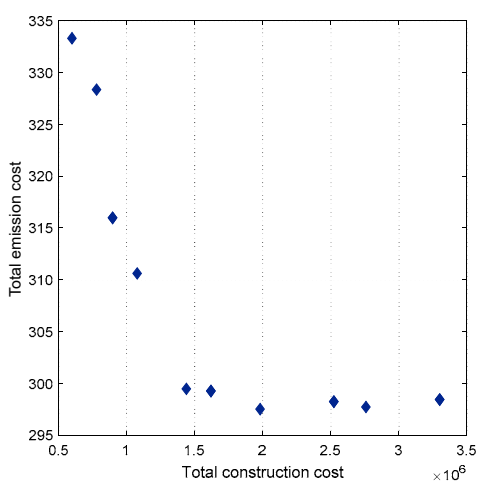

(d)

Figure 13. Pareto fronts of the Nguyen-Dupuis network (a) and the relation between the three goals in pairs (b-d).

The uncertain relation between the total emission cost and total travel cost reveals that improving the transportation efficiency does not necessarily lead to sustainable transportation development. To achieve the multi-objective optimization of the transportation system, we need to weigh the goals according to different traffic conditions.

\subsection{Sioux Falls Network}

To further test the proposed model and algorithm, we implemented the model in the Sioux Falls network (Figure A1), consisting of 24 nodes, 76 regular links (Table A1). We assume that the capacity of the regular lane is $2000 \mathrm{veh} / \mathrm{h}$, and the dedicated CAV lane is twice as much. Table A2 presents the total OD demand, in which the penetration rate of 
CAVs is assumed to be $50 \%$. Table 4 and Figure 14 show results in three situations in the Sioux Falls network. More specifically, compared to scenario 1, scenario 2 could reduce total emission costs by $10 \%$. Compared with scenario 1 and scenario 2 , scenario 3 could reduce the total travel time costs by $8.81 \%$ and $8.81 \%$, respectively. Similarly, the total emission cost was reduced by $17.4 \%$ and $8.25 \%$, respectively. Table 5 records the Pareto front solutions. The first column shows the solution number. The second column denotes the specific CAV lanes link location. The last three columns are the value of the objective.

Table 4. Comparison results in the two scenarios in the Sioux Falls network.

\begin{tabular}{cccc}
\hline & \multicolumn{3}{c}{ Average of Pareto Front } \\
\cline { 2 - 4 } & Total Travel Time Cost (USD 10 & Total Emission Cost (USD 000) & Total Construction Cost (USD 10 \\
\hline Scenario 1 & 1.28 & 9.32 & - \\
Scenario 2 & 1.28 & 8.39 & - \\
Scenario 3 & 1.17 & 7.70 & 2.04 \\
\hline
\end{tabular}

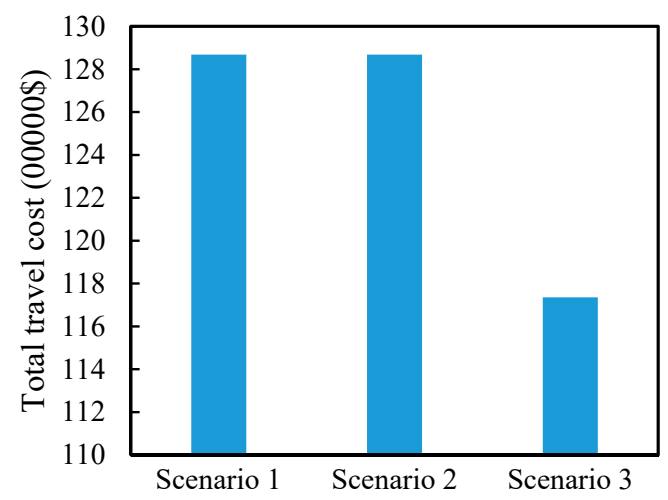

(a)

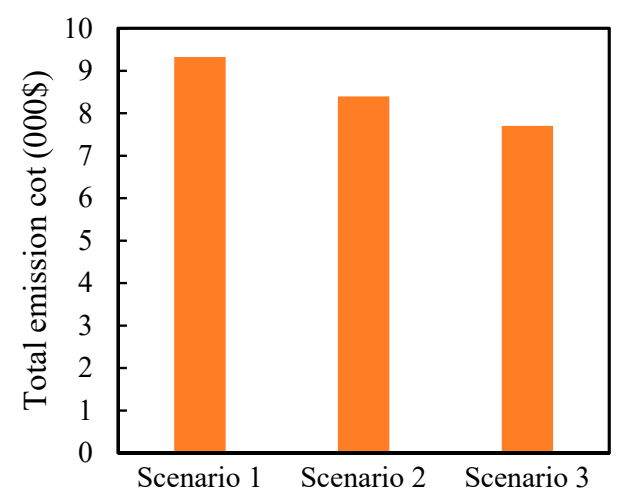

(b)

Figure 14. Total travel cost (a) and total emission cost (b) in Sioux Fall network.

Table 5. Pareto fronts solution of the Sioux Falls network.

\begin{tabular}{|c|c|c|c|c|}
\hline $\begin{array}{l}\text { Solution } \\
\text { Number }\end{array}$ & CAV Lanes Location & $\begin{array}{c}\text { Total Travel Cost } \\
\text { (USD) }\end{array}$ & $\begin{array}{l}\text { Total Emission } \\
\text { Cost (USD) }\end{array}$ & $\begin{array}{c}\text { Total Construction } \\
\text { Cost (USD 106) } \\
\end{array}$ \\
\hline 1 & $6 \rightarrow 8 ; 10 \rightarrow 11 ; 11 \rightarrow 10 ; 10 \rightarrow 15$ & $11,801,904$ & 8595.04 & 1 \\
\hline 2 & $5 \rightarrow 4 ; 6 \rightarrow 8 ; 8 \rightarrow 6 ; 10 \rightarrow 11 ; 11 \rightarrow 10 ; 10 \rightarrow 15$ & $11,747,267$ & 8561.43 & 1.7 \\
\hline 3 & $5 \rightarrow 4 ; 6 \rightarrow 8 ; 8 \rightarrow 6 ; 10 \rightarrow 11 ; 11 \rightarrow 10 ; 11 \rightarrow 14 ; 15 \rightarrow 10 ; 15 \rightarrow 14 ; 9 \rightarrow 5$ & $11,703,946$ & 8534.00 & 2.6 \\
\hline 4 & $4 \rightarrow 5 ; 5 \rightarrow 4 ; 6 \rightarrow 8 ; 8 \rightarrow 6 ; 14 \rightarrow 15$ & $11,785,825$ & 8583.64 & 1.1 \\
\hline 5 & $4 \rightarrow 5 ; 5 \rightarrow 4 ; 6 \rightarrow 8 ; 8 \rightarrow 6 ; 14 \rightarrow 15 ; 15 \rightarrow 14$ & $11,751,644$ & 8562.29 & 1.4 \\
\hline 6 & $4 \rightarrow 5 ; 5 \rightarrow 4 ; 6 \rightarrow 8 ; 8 \rightarrow 6 ; 14 \rightarrow 15 ; 15 \rightarrow 14 ; 5 \rightarrow 9$ & $11,736,848$ & 8553.13 & 1.9 \\
\hline 7 & $4 \rightarrow 5 ; 5 \rightarrow 4 ; 6 \rightarrow 8 ; 8 \rightarrow 6 ; 11 \rightarrow 14 ; 14 \rightarrow 15 ; 15 \rightarrow 14$ & $11,745,921$ & 8560.24 & 1.8 \\
\hline 8 & $4 \rightarrow 5 ; 5 \rightarrow 4 ; 6 \rightarrow 8 ; 8 \rightarrow 6 ; 10 \rightarrow 11 ; 14 \rightarrow 15 ; 15 \rightarrow 14$ & $11,750,838$ & 8561.72 & 1.5 \\
\hline 9 & $4 \rightarrow 5 ; 5 \rightarrow 4 ; 6 \rightarrow 8 ; 8 \rightarrow 6 ; 10 \rightarrow 11 ; 15 \rightarrow 10 ; 14 \rightarrow 15 ; 15 \rightarrow 14$ & $11,723,662$ & 8544.17 & 2.1 \\
\hline 10 & $4 \rightarrow 5 ; 5 \rightarrow 4 ; 6 \rightarrow 8 ; 8 \rightarrow 6 ; 10 \rightarrow 11 ; 15 \rightarrow 10 ; 14 \rightarrow 15 ; 15 \rightarrow 14 ; 5 \rightarrow 9$ & $11,705,760$ & 8532.51 & 2.6 \\
\hline 11 & $4 \rightarrow 5 ; 5 \rightarrow 4 ; 6 \rightarrow 8 ; 8 \rightarrow 6 ; 10 \rightarrow 11 ; 11 \rightarrow 14 ; 15 \rightarrow 10 ; 15 \rightarrow 14 ; 5 \rightarrow 9$ & $11,701,989$ & 8530.01 & 2.7 \\
\hline 12 & $4 \rightarrow 5 ; 5 \rightarrow 4 ; 6 \rightarrow 8 ; 8 \rightarrow 6 ; 11 \rightarrow 10 ; 11 \rightarrow 14 ; 15 \rightarrow 10 ; 14 \rightarrow 15 ; 15 \rightarrow 14$ & $11,709,023$ & 8534.33 & 2.5 \\
\hline 13 & $4 \rightarrow 5 ; 5 \rightarrow 4 ; 6 \rightarrow 8 ; 8 \rightarrow 6 ; 11 \rightarrow 10 ; 11 \rightarrow 14 ; 15 \rightarrow 10 ; 14 \rightarrow 15 ; 15 \rightarrow 14$ & $11,695,793$ & 8525.90 & 3 \\
\hline
\end{tabular}

Figure 15 represents the emission cost and total energy consumption in the Sioux Fall network. Figure 15a presents the change of three pollutants for the three scenarios. Specifically, compared to scenario 1 and scenario 2, the CO decreases by $17.5 \%$ and $8.3 \%$ in scenario 3, respectively. Similarly, the NOx is reduced by $16.2 \%$ and $7.1 \%$, respectively; VOC are reduced by $17.4 \%$ and $8.3 \%$, respectively. Figure $15 \mathrm{~b}$ shows the result of the energy consumption and $\mathrm{CO}_{2}$ emission for the three scenarios. In further detailed, compared to scenario 1 and scenario 2, the total energy consumption decreased by $10.3 \%$ and $0.8 \%$ in scenario 3, respectively. Similarly, the total $\mathrm{CO}_{2}$ emission could be reduced by $10.2 \%$ and $0.9 \%$, respectively. 


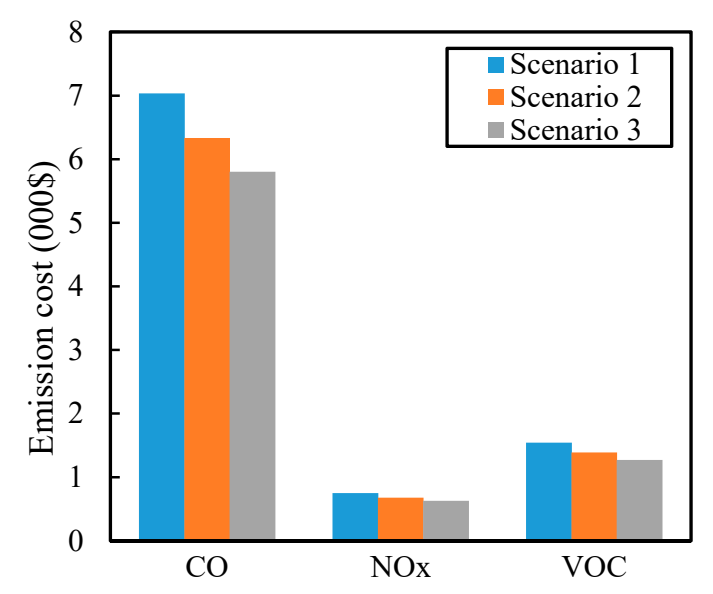

(a)

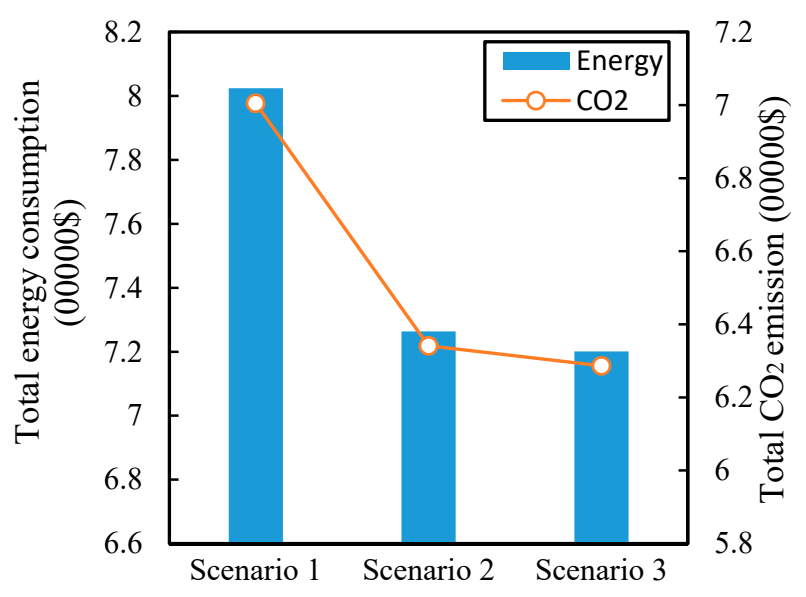

(b)

Figure 15. Emission cost (a) and total energy consumption (b) in the Sioux Fall network.

The above analysis further demonstrates that the model and algorithm proposed in this paper are effective and efficient.

\section{Conclusions}

We developed a novel dedicated CAV lanes optimal design problem with practical significance and environmental considerations in this work. The vehicle emission as an optimization objective was introduced. We established a multi-objective bi-level programming model with multi-class network equilibrium constraints for the proposed problem. The upper level involves implementing dedicated CAV lanes under multi-objective value, and the lower-level determines the equilibrium flow. The equilibrium constraints are formulated by a set of nonlinear complementarity constraints that characterize the vehicle choice and route choice behavior. To solve the proposed dedicated CAV lane optimal design problem, we propose an integrated solution framework that integrates the meta-heuristic algorithm, diagonalized algorithm, and Frank-Wolfe algorithm. Finally, two numerical experiments demonstrate the effectiveness of the proposed method. It was found that the proposed dedicated CAV lanes implementation program contributes to reduce the network travel time and emission.

We implemented the model and solution algorithm in two classic network cases, which illustrates the efficiency and effectiveness. Several findings are obtained based on numerical examples. First, implementing dedicated CAV lanes could reduce the system total time cost, total emission cost, and total energy consumption by about $2.03 \%, 10.42 \%$, and $9.4 \%$ in the Nguyen-Dupuis network. Second, implementing the dedicated CAV lanes could further reduce the total travel cost and emission cost with CAV market penetration evolution. Third, as a dedicated CAV lane is expected to have greater capacity than a regular lane, the system performance will also benefit from the overall capacity increase by implementing dedicated CAV lanes. For example, when CAV market penetration is $50 \%$, a three-fold capacity increase by converting a regular lane to a dedicated CAV lane would reduce the total travel cost and total emission cost by $5.5 \%$ and $4.6 \%$, respectively, compared to a two-fold capacity increase. Finally, we found that minimizing the total travel time cost and minimizing the total emission cost are not necessarily always aligned, and can be affected by vehicle travel speeds. Overall, this research provides a modeling and analyzing framework that can be used to inform planning and decision-making for dedicated CAV lane implementation in future urban transportation networks. The revealed Pareto relationship between construction cost and system performance offers insights into the potential returns of investment under the limited budget. The emission and energy use analysis presented in this paper further lends support to assessing the environmental and energy impact of CAV use and improve policies related to CAV infrastructure development. 
There are some future research issues worth investigating. First, we can focus on the CAV demand evolution study. For example, we can establish an applicable CAV forecasting model to capture the CAV demand change. Second, it is meaningful to consider lane capacity change with the CAV market penetration to improve model accuracy. The relationship between the lane capacity and CAV market penetration needs more discussion. Finally, it is useful to study another effective method to solve the model. For instance, the decomposition algorithms and linearization-based solution approach.

Author Contributions: Conceptualization, Y.L.; data curation, Y.L.; formal analysis, Y.L., and G.W.; funding acquisition, Y.L. and R.W.; investigation, Y.L. and J.T.; methodology, Y.L.; supervision, H.J. and B.Z.; writing—original draft, Y.L.; writing—review and editing, Y.L., B.Z. and H.M. All authors have read and agreed to the published version of the manuscript.

Funding: This research was funded by the National Natural Science Foundation of China [grant number 52072143].

Institutional Review Board Statement: Not applicable.

Informed Consent Statement: Not applicable.

Data Availability Statement: The data presented in this study are available on request from the corresponding author. The data are not publicly available due to privacy.

Acknowledgments: Thank you to all reviewers for their suggestions to make this paper better.

Conflicts of Interest: We declare that we have no conflict of interest.

\section{Appendix A}

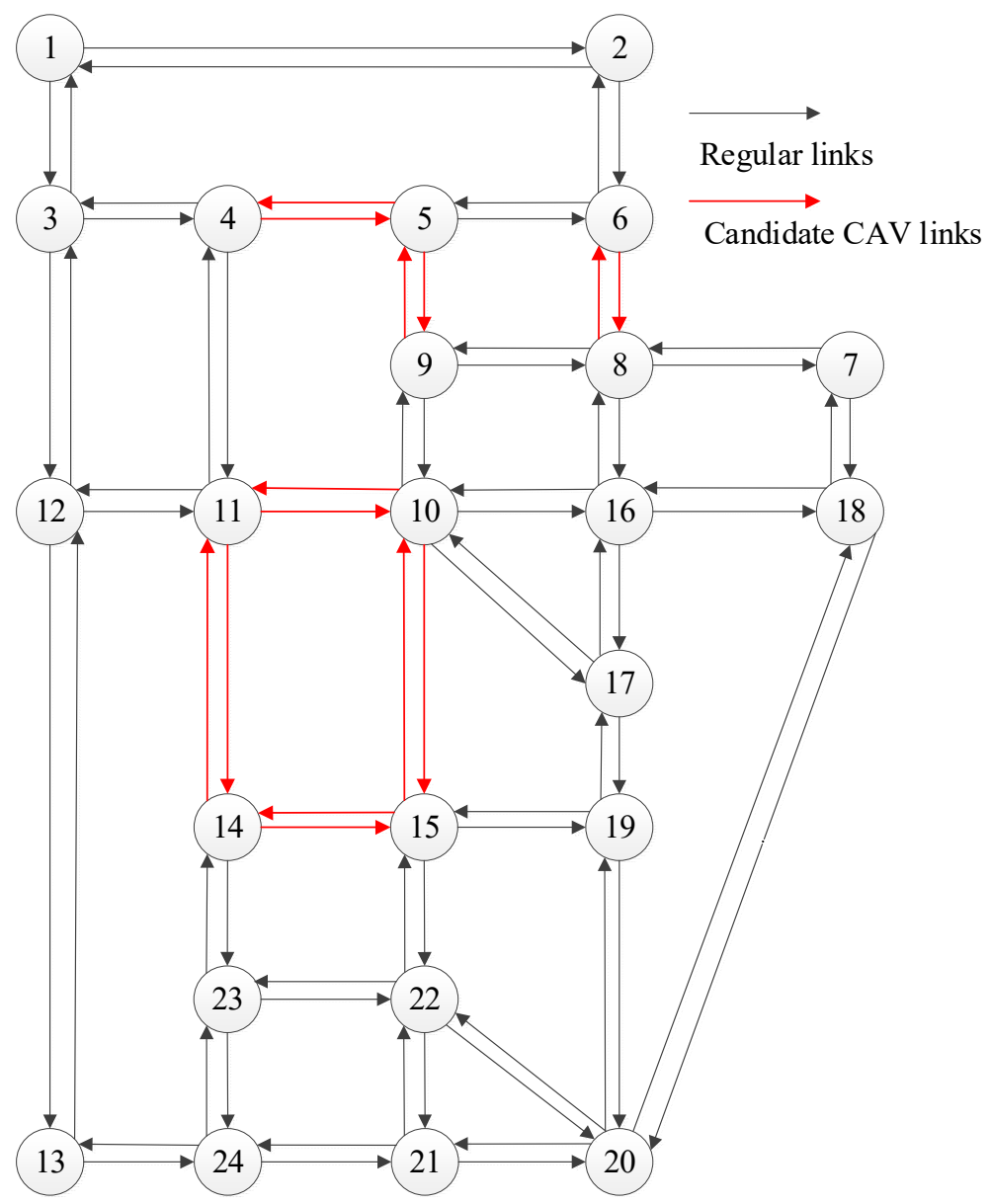

Figure A1. The Sioux Fall network. 


\section{Appendix B}

Table A1. Link characteristics of the Sioux Falls network.

\begin{tabular}{|c|c|c|c|c|c|c|c|}
\hline Link & $\begin{array}{l}\text { Time } \\
\text { (min) }\end{array}$ & Length & $\begin{array}{c}\text { Initial } \\
\text { Capacity } \\
\text { (veh/h) }\end{array}$ & Link & $\begin{array}{l}\text { Time } \\
(\mathrm{min})\end{array}$ & Length & $\begin{array}{c}\text { Initial } \\
\text { Capacity } \\
\text { (veh/h) }\end{array}$ \\
\hline $1-2$ & 6 & 6 & 10,000 & $13-24$ & 4 & 4 & 6000 \\
\hline $1-3$ & 4 & 4 & 10,000 & $14-11$ & 4 & 4 & 4000 \\
\hline $2-1$ & 6 & 6 & 10,000 & $14-15$ & 3 & 3 & 6000 \\
\hline $2-6$ & 8 & 8 & 6000 & $14-23$ & 4 & 4 & 4000 \\
\hline $3-1$ & 4 & 4 & 10,000 & $15-10$ & 6 & 6 & 8000 \\
\hline $3-4$ & 4 & 4 & 8000 & $15-14$ & 3 & 3 & 6000 \\
\hline 3-12 & 4 & 4 & 10,000 & $15-19$ & 3 & 3 & 8000 \\
\hline $4-3$ & 4 & 4 & 8000 & $15-22$ & 3 & 3 & 8000 \\
\hline $4-5$ & 2 & 2 & 8000 & $16-8$ & 5 & 5 & 6000 \\
\hline $4-11$ & 6 & 6 & 6000 & $16-10$ & 4 & 4 & 6000 \\
\hline $5-4$ & 2 & 2 & 8000 & $16-17$ & 2 & 2 & 6000 \\
\hline $5-6$ & 4 & 4 & 4000 & $16-18$ & 3 & 3 & 8000 \\
\hline $5-9$ & 5 & 5 & 6000 & $17-10$ & 8 & 8 & 4000 \\
\hline $6-2$ & 8 & 8 & 6000 & $17-16$ & 2 & 2 & 6000 \\
\hline $6-5$ & 4 & 4 & 4000 & $17-19$ & 2 & 2 & 4000 \\
\hline $6-8$ & 2 & 2 & 6000 & $18-7$ & 2 & 2 & 10,000 \\
\hline $7-8$ & 3 & 3 & 6000 & $18-16$ & 3 & 3 & 8000 \\
\hline $7-18$ & 2 & 2 & 10,000 & $18-20$ & 14 & 14 & 10,000 \\
\hline $8-6$ & 2 & 2 & 6000 & $19-15$ & 3 & 3 & 8000 \\
\hline $8-7$ & 3 & 3 & 6000 & $19-17$ & 2 & 2 & 4000 \\
\hline $8-9$ & 3 & 3 & 4000 & $19-20$ & 4 & 4 & 4000 \\
\hline $8-16$ & 5 & 5 & 6000 & $20-18$ & 14 & 14 & 10,000 \\
\hline $9-5$ & 5 & 5 & 6000 & $20-19$ & 4 & 4 & 4000 \\
\hline $9-8$ & 3 & 3 & 4000 & $20-21$ & 6 & 6 & 6000 \\
\hline $9-10$ & 3 & 3 & 8000 & $20-22$ & 5 & 5 & 6000 \\
\hline $10-9$ & 3 & 3 & 8000 & $21-20$ & 6 & 6 & 6000 \\
\hline $10-11$ & 1 & 1 & 6000 & $21-22$ & 2 & 2 & 6000 \\
\hline $10-15$ & 6 & 6 & 8000 & $21-24$ & 3 & 3 & 4000 \\
\hline $10-16$ & 4 & 4 & 6000 & $22-15$ & 3 & 3 & 8000 \\
\hline $10-17$ & 8 & 8 & 4000 & $22-20$ & 5 & 5 & 6000 \\
\hline $11-4$ & 6 & 6 & 6000 & $22-21$ & 2 & 2 & 6000 \\
\hline $11-10$ & 1 & 1 & 6000 & $22-23$ & 4 & 4 & 4000 \\
\hline $11-12$ & 6 & 6 & 6000 & $23-14$ & 4 & 4 & 4000 \\
\hline $11-14$ & 4 & 4 & 4000 & $23-22$ & 4 & 4 & 4000 \\
\hline $12-3$ & 4 & 4 & 10,000 & $23-24$ & 2 & 2 & 6000 \\
\hline $12-11$ & 6 & 6 & 6000 & $24-13$ & 4 & 4 & 6000 \\
\hline $12-13$ & 3 & 3 & 10,000 & $24-21$ & 3 & 3 & 4000 \\
\hline $13-12$ & 3 & 3 & 10,000 & $24-23$ & 2 & 2 & 6000 \\
\hline
\end{tabular}

Table A2. Total origin-destination (OD) demand of the Sioux Falls network (veh/h).

\begin{tabular}{cccccccc}
\hline O-D & Demand & O-D & Demand & O-D & Demand & O-D & Demand \\
\hline $1-6$ & 20,000 & $6-15$ & 12,000 & $11-1$ & 20,000 & $21-7$ & 4000 \\
$1-7$ & 20,000 & $6-21$ & 12,000 & $11-6$ & 12,000 & $21-11$ & 8000 \\
$1-11$ & 20,000 & $7-1$ & 20,000 & $11-7$ & 20,000 & $21-15$ & 12,000 \\
$1-15$ & 20,000 & $7-6$ & 8000 & $11-21$ & 8000 & $15-1$ & 20,000 \\
$1-21$ & 20,000 & $7-11$ & 20,000 & $11-15$ & 12,000 & $15-6$ & 12,000 \\
$6-1$ & 20,000 & $7-21$ & 16,000 & $21-1$ & 8000 & $15-7$ & 12,000 \\
$6-11$ & 12,000 & $7-15$ & 12,000 & $21-6$ & 12,000 & $15-11$ & 12,000 \\
\hline
\end{tabular}




\section{References}

1. Ma, X.; Jin, J.; Lei, W. Multi-Criteria Analysis of Optimal Signal Plans Using Microscopic Traffic Models. Transp. Res. Part D Transp. Environ. 2014, 32, 1-14. [CrossRef]

2. Banister, D. Sustainable transport: Challenges and opportunities. Transportmetrica 2007, 3, 91-106. [CrossRef]

3. Szeto, W.Y.; Jaber, X.; Wong, S.C. Road Network Equilibrium Approaches to Environmental Sustainability. Transp. Rev. 2012, 32, 491-518. [CrossRef]

4. Wang, Y.; Szeto, W.Y. Excessive Noise Paradoxes in Urban Transportation Networks. Transp. A Transp. Sci. 2017, 13, 195-221. [CrossRef]

5. Huang, W.; Xu, G.; Lo, H.K. Pareto-Optimal Sustainable Transportation Network Design under Spatial Queuing. Netw. Spat. Econ. 2020, 20, 637-673. [CrossRef]

6. Xu, X.; Chen, A.; Yang, C. A Review of Sustainable Network Design for Road Networks. KSCE J. Civ. Eng. 2016, 20, 1084-1098. [CrossRef]

7. Alawadhi, M.; Almazrouie, J.; Kamil, M.; Khalil, K.A. A Systematic Literature Review of the Factors Influencing the Adoption of Autonomous Driving. Int. J. Syst. Assur. Eng. Manag. 2020. [CrossRef]

8. Calvert, S.; Mahmassani, H.; Meier, J.-N.; Varaiya, P.; Hamdar, S.; Chen, D.; Li, X.; Talebpour, A.; Mattingly, S.P. Traffic Flow of Connected and Automated Vehicles: Challenges and Opportunities. In Road Vehicle Automation 4; Meyer, G., Beiker, S., Eds.; Springer International Publishing: Cham, Switzerland, 2018; pp. 235-245; ISBN 978-3-319-60933-1.

9. Han, X.; Ma, R.; Zhang, H.M. Energy-Aware Trajectory Optimization of CAV Platoons through a Signalized Intersection. Transp. Res. Part C Emerg. Technol. 2020, 118, 102652. [CrossRef]

10. Lu, G.; Nie, Y.; Liu, X.; Li, D. Trajectory-Based Traffic Management inside an Autonomous Vehicle Zone. Transp. Res. Part B Methodol. 2019, 120, 76-98. [CrossRef]

11. Talebpour, A.; Mahmassani, H.S. Influence of Connected and Autonomous Vehicles on Traffic Flow Stability and Throughput. Transp. Res. Part C Emerg. Technol. 2016, 71, 143-163. [CrossRef]

12. Gong, S.; Shen, J.; Du, L. Constrained Optimization and Distributed Computation Based Car Following Control of a Connected and Autonomous Vehicle Platoon. Transp. Res. Part B Methodol. 2016, 94, 314-334. [CrossRef]

13. Li, Z.; Elefteriadou, L.; Ranka, S. Signal Control Optimization for Automated Vehicles at Isolated Signalized Intersections. Transp. Res. Part C Emerg. Technol. 2014, 49, 1-18. [CrossRef]

14. Vahidi, A.; Sciarretta, A. Energy Saving Potentials of Connected and Automated Vehicles. Transp. Res. Part C Emerg. Technol. 2018, 95, 822-843. [CrossRef]

15. Fagnant, D.J.; Kockelman, K.M. The Travel and Environmental Implications of Shared Autonomous Vehicles, Using Agent-Based Model Scenarios. Transp. Res. Part C Emerg. Technol. 2014, 40, 1-13. [CrossRef]

16. Kalra, N.; Paddock, S.M. Driving to Safety: How Many Miles of Driving Would It Take to Demonstrate Autonomous Vehicle Reliability? Transp. Res. Part A Policy Pract. 2016, 94, 182-193. [CrossRef]

17. Conlon, J.; Lin, J. Greenhouse Gas Emission Impact of Autonomous Vehicle Introduction in an Urban Network. Transp. Res. Rec. 2019, 2673, 142-152. [CrossRef]

18. Miao, H.; Jia, H.; Li, J.; Qiu, T.Z. Autonomous Connected Electric Vehicle (ACEV)-Based Car-Sharing System Modeling and Optimal Planning: A Unified Two-Stage Multi-Objective Optimization Methodology. Energy 2019, 169, 797-818. [CrossRef]

19. Chen, D.; Ahn, S.; Chitturi, M.; Noyce, D.A. Towards Vehicle Automation: Roadway Capacity Formulation for Traffic Mixed with Regular and Automated Vehicles. Transp. Res. Part B Methodol. 2017, 100, 196-221. [CrossRef]

20. Chen, Z.; He, F.; Zhang, L.; Yin, Y. Optimal Deployment of Autonomous Vehicle Lanes with Endogenous Market Penetration. Transp. Res. Part C Emerg. Technol. 2016, 72, 143-156. [CrossRef]

21. Chen, S.; Wang, H.; Meng, Q. Designing Autonomous Vehicle Incentive Program with Uncertain Vehicle Purchase Price. Transp. Res. Part C Emerg. Technol. 2019, 103, 226-245. [CrossRef]

22. Milakis, D.; van Arem, B.; van Wee, B. Policy and Society Related Implications of Automated Driving: A Review of Literature and Directions for Future Research. J. Intell. Transp. Syst. 2017, 21, 324-348. [CrossRef]

23. Bagloee, S.A.; Sarvi, M.; Patriksson, M.; Rajabifard, A. A Mixed User-Equilibrium and System-Optimal Traffic Flow for Connected Vehicles Stated as a Complementarity Problem. Comput. Aided Civ. Infrastruct. Eng. 2017, 32, 562-580. [CrossRef]

24. Levin, M.W.; Boyles, S.D. A Cell Transmission Model for Dynamic Lane Reversal with Autonomous Vehicles. Transp. Res. Part C Emerg. Technol. 2016, 68, 126-143. [CrossRef]

25. Zhang, K.; Nie, Y. (Marco) Mitigating the Impact of Selfish Routing: An Optimal-Ratio Control Scheme (ORCS) Inspired by Autonomous Driving. Transp. Res. Part C Emerg. Technol. 2018, 87, 75-90. [CrossRef]

26. Jiang, N. Optimal Signal Design for Mixed Equilibrium Networks with Autonomous and Regular Vehicles. Available online: https: / / www.hindawi.com/journals/jat/2017/5649823/ (accessed on 25 February 2020).

27. Chen, Z.; Lin, X.; Yin, Y.; Li, M. Path Controlling of Automated Vehicles for System Optimum on Transportation Networks with Heterogeneous Traffic Stream. Transp. Res. Part C Emerg. Technol. 2020, 110, 312-329. [CrossRef]

28. Adornato, B.; Patil, R.; Filipi, Z.; Baraket, Z.; Gordon, T. Characterizing Naturalistic Driving Patterns for Plug-in Hybrid Electric Vehicle Analysis. In Proceedings of the 2009 IEEE Vehicle Power and Propulsion Conference, Dearborn, MI, USA, 7-10 September 2009; pp. 655-660. 
29. Liang, Y.; Lee, J.D.; Yekhshatyan, L. How Dangerous Is Looking Away From the Road? Algorithms Predict Crash Risk From Glance Patterns in Naturalistic Driving. Hum. Factors 2012, 54, 1104-1116. [CrossRef]

30. Rahwan, I.; Cebrian, M.; Obradovich, N.; Bongard, J.; Bonnefon, J.-F.; Breazeal, C.; Crandall, J.W.; Christakis, N.A.; Couzin, I.D.; Jackson, M.O.; et al. Machine Behaviour. Nature 2019, 568, 477-486. [CrossRef]

31. Bonnefon, J.-F.; Shariff, A.; Rahwan, I. The Social Dilemma of Autonomous Vehicles. Science 2016, 352, 1573-1576. [CrossRef] [PubMed]

32. Balsa-Barreiro, J.; Ambuühl, L.; Menéndez, M.; Pentland, A. Mapping Time-Varying Accessibility and Territorial Cohesion with Time-Distorted Maps. IEEE Access 2019, 7, 41702-41714. [CrossRef]

33. Sharma, S.; Mathew, T.V. Multiobjective Network Design for Emission and Travel-Time Trade-off for a Sustainable Large Urban Transportation Network. Environ. Plan. B Plan. Des 2011, 38, 520-538. [CrossRef]

34. Yin, Y.; Lawphongpanich, S. Internalizing Emission Externality on Road Networks. Transp. Res. Part D Transp. Environ. 2006, 11, 292-301. [CrossRef]

35. Szeto, W.Y.; Lo, H.K. Transportation Network Improvement and Tolling Strategies: The Issue of Intergeneration Equity. Transp. Res. Part A Policy Pract. 2006, 40, 227-243. [CrossRef]

36. Chen, Z.; He, F.; Yin, Y.; Du, Y. Optimal Design of Autonomous Vehicle Zones in Transportation Networks. Transp. Res. Part B Methodol. 2017, 99, 44-61. [CrossRef]

37. Liu, Z.; Song, Z. Strategic Planning of Dedicated Autonomous Vehicle Lanes and Autonomous Vehicle/Toll Lanes in Transportation Networks. Transp. Res. Part C Emerg. Technol. 2019, 106, 381-403. [CrossRef]

38. Chen, X.; Lin, X.; He, F.; Li, M. Modeling and Control of Automated Vehicle Access on Dedicated Bus Rapid Transit Lanes. Transp. Res. Part C Emerg. Technol. 2020, 120, 102795. [CrossRef]

39. Tientrakool, P.; Ho, Y.-C.; Maxemchuk, N.F. Highway Capacity Benefits from Using Vehicle-to-Vehicle Communication and Sensors for Collision Avoidance. In Proceedings of the 2011 IEEE Vehicular Technology Conference (VTC Fall), San Francisco, CA, USA, 5-8 September 2011; pp. 1-5.

40. Penic, M.A.; Upchurch, J. TRANSYT-7F: Enhancement for fuel consumption, pollution emissions, and user costs. J. Transp. Res. Board 1992, 1360, 104-111.

41. US Bureau of Public Roads. Traffic Assignment Manual for Application with a Large, High Speed Computer; U.S. Department of Commerce, Bureau of Public Roads, Office of Planning, Urban Planning Division: Washington, DC, USA, 1964.

42. Wardrop, J.G.; Whitehead, J.I. Correspondence. Some Theoretical Aspects of Road Traffic Research. Proc. Inst. Civ. Eng. 1952, 1, 767-768. [CrossRef]

43. Yang, H.; Huang, H.-J. The Multi-Class, Multi-Criteria Traffic Network Equilibrium and Systems Optimum Problem. Transp. Res. Part B Methodol. 2004, 38, 1-15. [CrossRef]

44. Deb, K.; Pratap, A.; Agarwal, S.; Meyarivan, T. A Fast and Elitist Multiobjective Genetic Algorithm: NSGA-II. IEEE Trans. Evol. Comput. 2002, 6, 182-197. [CrossRef]

45. Frank, M.; Wolfe, P. An algorithm for quadratic programming. Nav. Res. Logist. Q. 1956, 3, 95-110. [CrossRef]

46. Abdulaal, M.; LeBlanc, L.J. Methods for Combining Modal Split and Equilibrium Assignment Models. Transp. Sci. 1979. [CrossRef]

47. Daganzo, C.F.; Sheffi, Y. On Stochastic Models of Traffic Assignment. Transp. Sci. 1977, 11, 253-274. [CrossRef]

48. Wang, Y.; Szeto, W.Y. Multiobjective Environmentally Sustainable Road Network Design Using Pareto Optimization: Environmentally Sustainable Road Network Design Using Pareto Optimization. Comput. Aided Civ. Infrastruct. Eng. 2017, 32, 964-987. [CrossRef] 